\title{
Linear canonical transformations and quantum phase: a unified canonical and algebraic approach
}

\author{
T Hakioglu \\ Physics Department, Bilkent University, Ankara 06533 Turkey \\ Received 25 September 1998, in final form 15 February 1999
}

\begin{abstract}
The algebra of generalized linear quantum canonical transformations is examined in the perspective of Schwinger's unitary-canonical operator basis. Formulation of the quantum phase problem within the theory of quantum canonical transformations and in particular with the generalized quantum action-angle phase space formalism is established and it is shown that the conceptual foundation of the quantum phase problem lies within the algebraic properties of the canonical transformations in the quantum phase space. The representations of the Wigner function in the generalized action-angle unitary operator pair for certain Hamiltonian systems with dynamical symmetry is examined. This generalized canonical formalism is applied to the quantum harmonic oscillator to examine the properties of the unitary quantum phase operator as well as the action-angle Wigner function.
\end{abstract}

\section{Introduction and review}

The quantum mechanical operator realization of the classical phase observable, well known as the historical quantum phase problem, is one of the oldest problems in quantum mechanics. In the quest for a correspondence between the classical action-angle (AA) variables and their quantum counterparts, Born et al investigated [1] the problem in the earliest days of quantum mechanics from the general perspective of building a theory of quantum canonical transformations and their unitary representations. The search for a quantum phase operator within this canonical perspective was specifically begun in one of Dirac's early works [2] in 1927 where the principal motivation was to extend the principle of correspondence to that between classical AA variables and their quantum counterparts. The quantum phase problem was then followed by the works of Heitler [3] and Louisell [4] where it was examined in terms of the quantization of the electromagnetic field. The introduction of trigonometric Hermitian phase operators by Susskind and Glogower [5] created a trigonometric approach to the phase problem. At this point, a landmark was reached with the introduction of a coherent state formalism by Glauber [6] and, with the development of laser physics in the 1960s, the theoretical and experimental investigation of the properties of the quantum phase became mainstreaminquantumoptics. Ontheotherhand, contemporarytoGlauber'swork, Carruthers and Nieto in their seminal paper [7], wisely entitled as Phase and angle variables in quantum mechanics, revived interest in the canonical approach advocated in the early days of quantum mechanics. Since our interest in this paper is within the canonical perspective, we refer the interested reader to, for instance, some recent reviews on the quantum phase as seen from the 
perspective of quantum optics [8,9]. In the search for the quantum counterpart of the classical AA pair, the canonical perspective in the quantum phase problem was furthered mainly by

the works of Rocca and Siruge [10], Boyer and Wolf [11], Moshinsky and Seligman [12], as well as Luis and Sanchez-Soto [13] and more recently by Lewis et al [14]. In an earlier paper [15], we introduced a different canonical-algebraic approach to the quantum phase problemfromthosein[11-14]bystartingfromthegeneralizeddiscreteunitary-cyclicfinite(D)

dimensional representations of the quantum phase space (QPS) distribution functions in terms ofSchwinger'soperatorbasis[16,17]. Therearetwocrucialpropertiesoftheserepresentations from the quantum phase operator point of view. The first one is that Schwinger's operator basis supports discrete cyclic finite D-dimensional subalgebraic representations with nonnegative normsintheD-dimensionalHilbertspace $\mathrm{H}_{\mathrm{D}}$.

Thesecyclicandadmissiblerepresentationsare

knowntobecrucialfortheexistenceofthephaseoperatorinanarbitrarybutfinite-dimensional algebra. On the other hand, the second crucial property is connected with the fact that the complete set of elements of the discrete finite-dimensional cyclic Schwinger operator basis are the generalized dual representations of the standard Wigner-Kirkwood (WK) [18] ones of the QPS [15-17]. Moreover, these elements are the generators of the discrete area preserving diffeomorphism on the two-dimensional toroidal lattice $\mathrm{ZD}_{\mathrm{D}} \times \mathrm{ZD}_{\mathrm{D}}$ which are known to respect the Fairlie-Fletcher-Zachos (FFZ) sine algebra [19]. As the dimension D is extended to infinity, a limit to continuum can be realized where the connection with Arnold's infinitesimal area preserving diffeomorphism [20] on the continuous two-torus is established. Hence, the representations of the QPS in terms of Schwinger's unitary-canonical operator basis paves a direct route to the algebraic formulation of the quantum phase operator in connection with the linear quantum canonical transformations (LCT). By this argument we imply that the algebraic formulation of the quantum phase problem is connected, through the Wigner-Weyl- Moyal (WWM) correspondence, with the existence of a canonical formalism of the quantum AA operators in the QPS. This correspondence, although it will be shown to be manifest for arbitrary but finite dimensions leading to the finite-dimensional algebraic realizations of the AA Wigner function, yields the desired correspondence between the quantum and the classical AA formalisms only in the transition to the continuum limit.

The main purpose of this paper is to extend the canonical-algebraic approach to the quantum phase problem in [15] by formulating this correspondence explicitly in terms of the generators of the LCT. The quantum AA operators will be found in terms of the generators of the LCT and it will be shown that the angle operator unitary-canonical to the quantum action will be identified as the unitary quantum phase operator.

Here we review some relevant parts of [15] for completeness. Some additional material is also included in the appendix. The duality relations between the discrete generalized WK phase space operator basis $1(\mathrm{n}) \mathrm{E}$ and the Schwinger operator basis $\mathrm{S}^{\wedge} \mathrm{mE}$ can be expressed as

$$
\hat{\Delta}(\vec{n})=\frac{1}{D^{3 / 2}} \sum_{\vec{m}} \mathrm{e}^{-\mathrm{i} \gamma_{0}(\vec{m} \times \vec{n})} \hat{S}_{\vec{m}} \hat{S}_{\vec{m}}=\frac{1}{\sqrt{D}} \sum_{\vec{n}} \mathrm{e}^{\mathrm{i} \gamma_{0}(\vec{m} \times \vec{n})} \hat{\Delta}(\vec{n})
$$

where $\mathrm{mE}=\left(\mathrm{m}_{1}, \mathrm{~m}_{2}\right), \mathrm{nE}=\left(\mathrm{n}_{1}, \mathrm{n}_{2}\right)$ are vectors in $\mathrm{ZD} \times \mathrm{ZD} ; \mathrm{mE} \times \mathrm{En} \equiv\left(\mathrm{m}_{1} \mathrm{n}_{2}-\mathrm{m}_{2} \mathrm{n}_{1}\right), \gamma_{0}=$ $2 \pi / \mathrm{D}$, withD describingthedimensionofthecyclicrepresentations. HeretheSchwinger operator 
basis $\mathrm{S}^{\wedge} \mathrm{mE}$ is defined in terms of a finite-dimensional unitary cyclic operator pair $\left(\mathrm{U}^{\wedge}, \mathrm{V}^{\wedge}\right)$ such

that

$$
\mathrm{U}_{{ }_{\mathrm{m} 1 \mathrm{~V}} \mathrm{~V}^{\wedge} \mathrm{m}_{2}=\mathrm{ei \gamma om}_{\mathrm{m} 2} \mathrm{~V}^{\wedge}{ }_{\mathrm{m} 2} \mathrm{U}^{\wedge} \mathrm{m} 1} \quad \hat{S}_{\vec{m}}=\mathrm{e}^{-\mathrm{i} \gamma_{0} m_{1} m_{2} / 2} \hat{\mathcal{U}}^{m_{1}} \hat{\mathcal{V}}^{m_{2}} .
$$

The D-dimensional cyclic eigenspace $\left\{\mid v_{i k}\right\}_{06 k 6(\mathrm{D}-1)}$ and $\left\{\mid \mathrm{uik}_{\mathrm{k}}\right\}_{06 \mathrm{k} 6(\mathrm{D}-1)}$ of the operators $\mathrm{U}^{\wedge}, \mathrm{V}^{\wedge}$ satisfy

$$
\begin{aligned}
& \mathrm{U}^{\wedge} \mid \text { vik }=\text { eiriorko }|\mathrm{kvik} \quad| \mathrm{vik}+\mathrm{D}=\mid \mathrm{vik} \\
& \mathrm{V}^{\wedge} \mid \text { uik }=\mathrm{e}-\quad \text { |uik } \quad \text { |uik+D }=\text { |uik } \\
& \mathrm{U}^{\wedge}|u \mathrm{uik}=| \mathrm{uik}_{\mathrm{k}} \mathrm{V}^{\wedge} \mid \text { vik } \\
& =\mid v i k+1
\end{aligned}
$$

and define a unitary Fourier duality as

$\{\mid$ vi $\}=\mathrm{F}^{\wedge}\{\mid$ ui $\} \quad$ where $\quad(\hat{\mathcal{F}})_{k, k^{\prime}}=\frac{1}{\sqrt{D}} \mathrm{e}^{-\mathrm{i} \gamma_{0} k k^{\prime}} \quad \hat{\mathcal{F}}^{\dagger}=\hat{\mathcal{F}}^{-1} \quad$ (4) where the dual

picture implies a Fourier automorphism on $\mathrm{U}^{\wedge}$ and $\mathrm{V}^{\wedge}$ in a sequence of transformations as

$$
\left(\begin{array}{c}
\hat{\mathcal{U}} \\
\hat{\mathcal{V}}
\end{array}\right) \stackrel{\hat{\mathcal{F}}}{\longrightarrow}\left(\begin{array}{c}
\hat{\mathcal{V}} \\
\hat{\mathcal{U}}^{-1}
\end{array}\right) \stackrel{\hat{\mathcal{F}}}{\longrightarrow}\left(\begin{array}{c}
\hat{\mathcal{U}}^{-1} \\
\hat{\mathcal{V}}^{-1}
\end{array}\right) \stackrel{\hat{\mathcal{F}}}{\longrightarrow}\left(\begin{array}{c}
\hat{\mathcal{V}}^{-1} \\
\hat{\mathcal{U}}
\end{array}\right) \stackrel{\hat{\mathcal{F}}}{\longrightarrow}\left(\begin{array}{c}
\hat{\mathcal{U}} \\
\hat{\mathcal{V}}
\end{array}\right) .
$$

It can be shown that this Fourier operator duality between $\mathrm{U}^{\wedge}$ and $\mathrm{V}^{\wedge}$ implies

$$
\hat{\mathcal{F}} \hat{S}_{\vec{m}} \hat{\mathcal{F}}^{-1}=\hat{S}_{R_{\pi / 2}: \vec{m}} \quad \hat{\mathcal{F}}^{4}=1 \quad \text { and } \quad R_{\pi / 2}^{4}=1
$$

where $\mathrm{R}_{\pi / 2}: \mathrm{mE}=\left(-\mathrm{m}_{2}, \mathrm{~m}_{1}\right)$ corresponds to a $\pi / 2$ rotation of the vector $\mathrm{mE}$ in the discrete phase space.

Equations (2) and (3) imply for the properties of the $\hat{S}_{\vec{m}}$ basis

$$
\begin{aligned}
& \mathrm{S}^{\wedge} \mathrm{m}^{\dagger} \mathrm{E}=\mathrm{S}^{\wedge}-\mathrm{mE} \\
& \mathrm{TrS}^{\wedge}{ }_{\mathrm{mE}}\left\{\mathrm{SS}^{\wedge}{ }^{\wedge m E}\right\}=\mathrm{eD} \delta \text { iromEm, E× Em0E o/2 } \mathrm{S}^{\wedge} \mathrm{mE}+\mathrm{mE0} \quad \text { (7) } \mathrm{E}=
\end{aligned}
$$

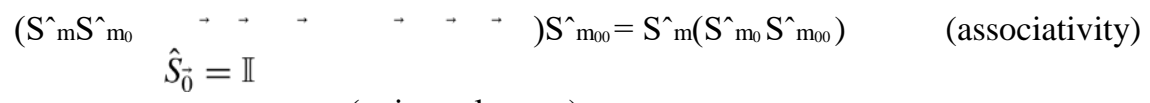

$$
\begin{aligned}
& \text { (unit element) } \\
& \mathrm{S}^{\wedge}{ }_{\mathrm{mE}} \mathrm{S}^{\wedge}{ }_{\mathrm{mE}}=\mathrm{I} \text { (inverse). }
\end{aligned}
$$

The generalized discrete Wigner function $\mathrm{W}(\mathrm{n}) \mathrm{E}$ in the physical state $\mid \psi \mathrm{i} \in \mathrm{H}_{\mathrm{D}}$ is defined by $[15,17]$

$$
\mathrm{W}(\mathrm{n}) \mathrm{E}=\mathrm{h} \psi\left|1\left({ }^{\wedge} \mathrm{n}\right) \mathrm{E}\right| \psi \mathrm{i}
$$


where equation (8) complies with all fundamental conditions that a generalized QPS distribution should satisfy. The normalization of equation (8) is based on an appropriate summation of the WK operator basis in equation (1) over the discrete phase space vector $\mathrm{nE}$. It is possible to use different normalizations when both (or one of the) labels are (is) continuous on the two-dimensional torus. In these particular cases the phase space representations are based on $\mathrm{R} \times \mathrm{R}$ or $\mathrm{Z} \times \mathrm{R}$ respectively. Different normalizations are necessary for different choices of the phase space variables in order to obtain the appropriate continuum limit for the Wigner function. For instance, the symmetric normalization is necessary when the discrete phase space labels approach to the continuous ones $\begin{array}{llllllllll}\text { simultaneously } & \text { (i.e. } & \mathrm{ZD} & \times & \mathrm{ZD} & \rightarrow & \mathrm{R} & \times & \mathrm{R} \text { ) }\end{array}$ suchasinthecaseofcanonicalpairofcoordinateandmomentumx,p leadingtothecontinuous phase space distribution $\mathrm{W}(\mathrm{x}, \mathrm{p})$. The limit to continuous AA Wigner function $\mathrm{W}(\mathrm{J}, \theta)$ is recovered [15] when one of the phase space labels is real and the other remains to be an integer in the limit $\mathrm{D} \rightarrow \infty$; hence $\mathrm{ZD}_{\mathrm{D}} \times \mathrm{ZD}_{\mathrm{D}} \rightarrow \mathrm{Z} \times \mathrm{R}$. In section 2 we base our formulation on the symmetric normalization as given in equation (1) whereas, in section 4 , the AA Wigner function is examined using the appropriate asymmetric normalization with $\mathrm{Z} \times \mathrm{R}$ without any loss of generality.

In section 2 we start the formulation of the LCT. Section 2.1 is devoted to the discrete scenario where the elements of LCT are in SL(2,ZD). Their action on Schwinger's discrete cyclic operator basis is defined. The conditions of existence of a unitary-canonical partner to the generators of LCT are found for an arbitrary Hilbert space dimension and, provided such conditions are met, the existence of the unitary-canonical partner for each irreducible representation is demonstrated in the strong operator sense.

In section 2.2 the continuous scenario is examined. The elements of the continuous LCT are examined within the context of the irreducible representations of SL(2,R). The operators corresponding to the unitary canonical partners of continuous LCT within each one parameter subgroup of $\operatorname{SL}(2, R)$ as well as the entire group are derived by the matrix elements of the diagonal representations of the corresponding group elements. Section 3 is devoted to the Hamiltonian system with its dynamical symmetry group corresponding to that of LCT. The unitary canonical partners to the generators of LCT are identified as the unitary phase operators and their equations of motion are derived separately for Hamiltonians with a continuous as well as discrete spectrum. The connections between the quantum AA formalism and the dynamical symmetry is established at the operator level. Section 4 is devoted to the construction of the AA Wigner function. The continuous scenario is treated in section 4.1 and the AA Wigner function of the generalized oscillator with a discrete cyclic spectrum is presented in 4.2. The limit to the quantum harmonic oscillator (QHO) AA formalism is also established.

\section{Generators of the linear canonical transformations}

$$
\text { 2.1. On the discrete toroidal lattice } \mathrm{ZD} \times \mathrm{ZD}
$$

The unitary Fourier automorphism in equation (5) implies the simplest discrete canonical transformation $\mathrm{mE} \rightarrow \mathrm{R}_{\pi / 2}: \mathrm{mE}$ on the phase space labels as given by equation (6). It was 
shown in [15] that equations (5) are a special case of a more general automorphic sequence produced by a unitary canonical transformation generator $\mathrm{G}^{\wedge}$ with $\mathrm{G}^{\wedge \dagger}=\mathrm{G}^{\wedge-1}$ where

$$
\begin{aligned}
& \mathrm{G}^{\wedge} \mathrm{U}^{\wedge} \mathrm{G}^{\wedge}=\mathrm{S}^{\wedge} \mathrm{sE} \quad \mathrm{G}^{\wedge} \mathrm{V}^{\wedge} \mathrm{G}^{\wedge}=\mathrm{S}^{\wedge}{ }_{\mathrm{tE}} \\
& \mathrm{U}^{\wedge} \longrightarrow \rightarrow \mathrm{G}^{\wedge} \mathrm{S}^{\wedge}{ }_{\mathrm{sE}}-\rightarrow \mathrm{G}^{\wedge} \mathrm{S}^{\wedge}{ }_{\mathrm{S} 1 \mathrm{EE}+\mathrm{S} 2 \mathrm{E}}-\longrightarrow{ }^{\cdots} \mathrm{G}^{\wedge} \\
& \mathrm{V}^{\wedge}-\rightarrow \mathrm{G}^{\wedge} \mathrm{S}^{\wedge}{ }_{\mathrm{tE}}-\rightarrow \mathrm{G}^{\wedge} \mathrm{S}^{\wedge}{ }_{\mathrm{t} 1 \mathrm{EE}+\mathrm{t} 2 \mathrm{E}}-\rightarrow{ }^{\cdots} \mathrm{G}^{\wedge} \quad .
\end{aligned}
$$

Such a unitary generator satisfies

$$
\hat{\mathcal{G}}^{\dagger} \hat{S}_{\vec{m}} \hat{\mathcal{G}}=\hat{S}_{R: \vec{m}} \quad \text { where } \quad \mathrm{R}: \mathrm{mE}=\mathrm{mE}^{0}=\left(\mathrm{s}_{1} \mathrm{~m}_{1}+\mathrm{t}_{1} \mathrm{~m}_{2}, \mathrm{~s}_{2} \mathrm{~m}_{1}+\mathrm{t}_{2} \mathrm{~m}_{2}\right)(10)
$$

with $\operatorname{det} R=\vec{s} \times \vec{t}=1$ where $\vec{s}=\left(s_{1}, s_{2}\right)$ and $\vec{t}=\left(t_{1}, t_{2}\right)$ are two arbitrary labelling vectors characterizing the $\mathrm{LCT}$ in $\mathrm{ZD}_{\mathrm{D}} \times \mathrm{ZD}$. Hence $\mathrm{R} \in \mathrm{SL}(2, \mathrm{ZD})$. Equations (5) and (6) correspond to a special realization of equation $(10)$ when $\mathrm{sE}=(0,1)$ and $\mathrm{tE}=(-1,0)$. The application of $\mathrm{G}^{\wedge}$ leaves equation (7) covariant.

Using equation (10) in equation (1) it can be shown that $\mathrm{G}^{\wedge}$ generates discrete canonical transformations in the WK basis as

$$
1\left(n E^{0}\right)=G^{\wedge} 1(n) E G^{\wedge}=1\left(R^{-1}: n\right) E
$$

where $\mathrm{R}^{-1}: \mathrm{nE}=\mathrm{nE}^{0}=\left(\mathrm{t}_{2} \mathrm{n}_{1}-\mathrm{t}_{1} \mathrm{n}_{2},-\mathrm{s}_{2} \mathrm{n}_{1}+\mathrm{s}_{1} \mathrm{n}_{2}\right)$.

The explicit form of $\mathrm{G}^{\wedge}$ and its irreducible representations have been studied in detail for the specific case of $\mathrm{D}$ being a prime of the type $\mathrm{D}=4 \mathrm{k} \pm 1$ where $\mathrm{k} \in \mathrm{Z}$, in connection with the Schwinger operator basis by regarding $G^{\wedge}$ as the generator of the time evolution (Hamiltonian) of the $\mathrm{SL}\left(2, \mathrm{Z}_{\mathrm{D}}\right)$ oscillator [21]. Specifically, $\mathrm{G}^{\wedge}$ has cyclic generators which can be chosen as

$$
g_{1}=\left(\begin{array}{ll}
1 & 1 \\
0 & 1
\end{array}\right) \quad g_{2}=\left(\begin{array}{cc}
g_{0} & 0 \\
0 & g_{0}^{-1}
\end{array}\right) \quad g_{3}=\left\{\left(\begin{array}{cc}
a & -b \\
b & a
\end{array}\right), a^{2}+b^{2}=1 \quad(\bmod D)\right\}
$$

with periods $D, D-$ ${ }^{D-1}=(\quad D)$ element of $Z_{D}$ where $g_{0} \quad 1 \quad \bmod \quad$.

For such D, the explicit form of $\mathrm{G}^{\wedge}$ satisfying equations (9), or more compactly equation (10), is given by [21] 


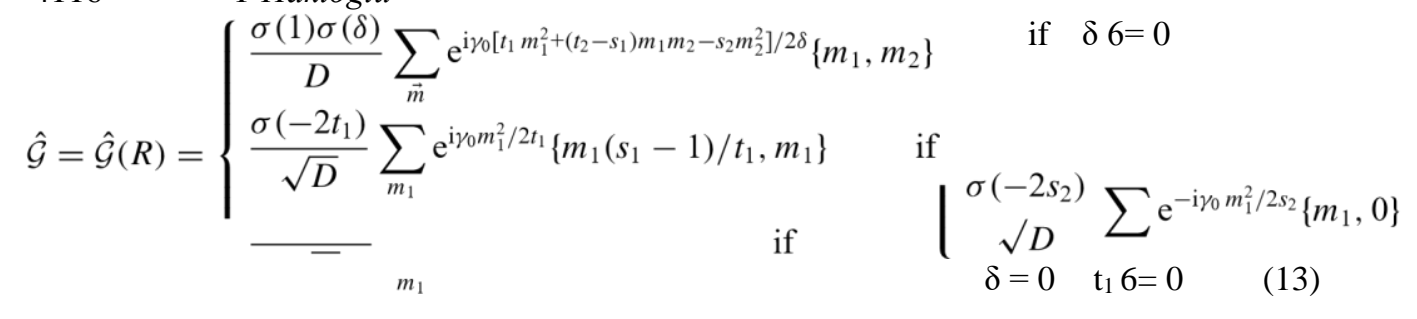

where it is defined that $\delta \equiv 2-\mathrm{s}_{1}-\mathrm{t}_{2}$ and $\left\{\mathrm{m}_{1}, \mathrm{~m}_{2}\right\} \equiv \mathrm{S}^{\wedge} \mathrm{mE}$. Here $\sigma(\mathrm{m})$ is the Gauss sum

$$
\equiv \frac{1}{\sqrt{D}} \sum_{n=0}^{D-1} \mathrm{e}^{\mathrm{i} \gamma_{0} m n^{2}} \sigma(\mathrm{m}) .
$$

It can be seen by direct inspection that $\mathrm{G}^{\wedge}\left(\mathrm{R}^{-1}\right)=\mathrm{G}^{\wedge-1}\left(\mathrm{R}^{)}=\mathrm{G}^{\wedge \dagger}(\mathrm{R})\right.$, namely $\mathrm{G}^{\wedge}$ is unitary.

Our main purpose in this section is to search for the condition of existence of a unitary canonical partner $\mathrm{O}^{\wedge}$ to $\mathrm{G}^{\wedge}$ such that

$$
\mathrm{G}^{\wedge} \mathrm{O}^{\wedge}=\bullet \mathrm{O}^{\wedge} \mathrm{G}^{\wedge} \quad|\bullet|=1 \quad\left[\mathrm{G}^{\wedge}, \bullet\right]=\left[\mathrm{O}^{\wedge}, \bullet\right]=0 .
$$

If equation (15) is satisfied for some pure phase factor $\bullet$ and a unitary $0^{\wedge}$, then we consider equation (15) as a generalized canonical commutation relation for the pair $\mathrm{G}^{\wedge}, \mathrm{O}^{\wedge}$. The equation (15) then implies that $\mathrm{O}^{\wedge}$ rotates the eigenspectrum of $\mathrm{G}^{\wedge}$ in a cyclic order and vice versa. It is beyond the scope of this paper to examine the characterization of the irreducible representations of the most general group defined by $\hat{\mathcal{G}}, \hat{\mathcal{O}}, \Omega$ in equation (15) above. Here, we confine our attention to those relatively simpler cases leading to the unique irreducible

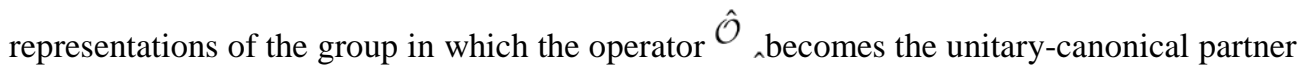
of the generalized canonical transformation generator G. For this purpose, let us start with the simplest case such that for some non-zero integers a,b,c we have

$$
\mathrm{G}^{\wedge \mathrm{a}}=\mathrm{O}^{\wedge \mathrm{b}}=\bullet^{\mathrm{c}}=1 .
$$

Thenwecallthegroupdefinedbytheelements $\mathrm{G}^{{ }^{\prime} 1} \mathrm{O}^{\wedge{ }^{\prime 2}} \bullet{ }^{3}$, where ${ }_{1},{ }_{2},{ }_{3}$ areintegersdefined (mod a), $(\bmod b),(\bmod c)$ respectively, as a discrete Heisenberg-Weyl group $0(a, b, c)$. An explicit calculation yields that the group elements are uniquely defined only when c divides both a and $b$ (i.e. $a^{a}=c a^{0}, b b=c c^{0}$ where $a^{0}, b^{0} \in Z$ ). Furthermore, from equations (16) and (15) we also have $\bullet=\boldsymbol{=}=\boldsymbol{=} 1$ which implies that a,b,c must have a greatest common divisor $\mathrm{d}$. These results imply that $\mathrm{a}=\mathrm{dc}^{00} \mathrm{a}^{0}, \mathrm{~b}=\mathrm{dc}^{00} \mathrm{~b}^{0}, \mathrm{c}=\mathrm{dc}^{00}$ where $\mathrm{c}^{00} \in \mathrm{Z}$. Without loss of generality we will assume that $\mathrm{c}^{00}=1$. The group defined by the Schwinger operator $\mathrm{m}^{1} \mathrm{~m}^{2} \mathrm{~m}^{3}$ basis $\mathrm{SmE}_{\mathrm{m}}$ in equations (2) and (3) with general elements as $\mathrm{U}^{\wedge} \mathrm{V}^{\wedge} \omega$ where $\mathrm{m}_{1}, \mathrm{~m}_{2}, \mathrm{~m}_{3}$ are integers ( $\left.\bmod \mathrm{D}\right)$ is then a specific example of $0(a, b, c)$ with $a=b=c=D$. The number of irreducible representations of $0(a, b, c)$ with $a=d a^{0}, b=d^{0}, c=d$ depends on the numbers $a^{0}, b^{0}$. For $a^{0}=$ 
$b^{0}=1$ there is only one irreducible representation which is d-dimensional and is given by the

Weyl matrices [22],

$$
\hat{\mathcal{G}} \rightarrow \operatorname{diag}\left(1, \Omega, \Omega^{2}, \ldots, \Omega^{d-1}\right) \quad \hat{\mathcal{O}} \rightarrow\left(\begin{array}{ccccc}
0 & 0 & \ldots & 0 & 1 \\
1 & 0 & \ldots & 0 & 0 \\
0 & 1 & 0 & \ldots & 0 \\
\vdots & \vdots & \vdots & \ddots & 0 \\
0 & 0 & \ldots & 1 & 0
\end{array}\right)
$$

where a unitarythe number of such irreducible representations is given by the product $0^{\wedge}$ satisfying equation (15) exists as given in equation (17). Foraob0 and they are alla $^{0}, \mathrm{~b}^{0} 6=1$,

d-dimensional. Up to a unitary equivalence, each irreducible representation is isomorphic to that in equation (17). The connection of direct product representation of $0(\mathrm{~d}, \mathrm{~d}, \mathrm{~d})$ and its connection with the Chinese remainder theorem for the unique prime factorization of $\mathrm{d}$ was studied recently in [23]. Each prime factor represents an independent physical degree of freedom allowing the extension of the phase space formalism presented here to more than one degrees of freedom $[16,17]$. A similar decomposition can also be performed for the more general group $0(\mathrm{a}, \mathrm{b}, \mathrm{d})$ with $\mathrm{a}, \mathrm{b}, \mathrm{d}$ as defined above. The correspondence between the discrete QPS AA formalism with one degree of freedom and the classical one can be extended to more than one degree of freedom at an algebraic level. Within the purpose of this paper we will establish this correspondence only for the case with one degree of freedom and examine the larger degrees of freedom in a separate paper.

There is already an extensive literature on the representations of the discrete canonical transformations induced by $\mathrm{G}^{\wedge}=\mathrm{SL}(2, \mathrm{ZD})$. One particularly important limit in the discrete scenario is when $\mathrm{G}^{\wedge}$ is represented only by the rotational generator ${ }_{1 / \mathrm{k}} \mathrm{g}_{3}$ in equation (12) ${ }_{4 \mathrm{k}}$ corresponding to the discrete fractional Fourier operator $\mathrm{F}^{\wedge}=\mathrm{G}^{\wedge}$ such that $\mathrm{G}^{\wedge}=\mathrm{I}$. This limit has been examined in detail both from the formal quantum mechanical [24] and more applied, non-algebraic perspectives [25, 26]. For an arbitrary Hilbert space dimension the multiplicities of the four distinct eigenvalues of the fractional Fourier operator ${ }_{1 / 4 \mathrm{k}} \mathrm{F}^{\wedge 1 / \mathrm{k}}$ are not identical [25] and neither $\mathrm{F}^{\wedge}$ nor $\mathrm{F}^{\wedge}$ has an exact unitary-canonical partner in the sense of $\mathrm{O}^{\wedge}$ satisfying equation (15).

\subsection{Generators of the infinitesimal canonical transformations in $\mathrm{R} \times \mathrm{R}$}

The modular group SL(2,ZD) does not have a proper continuous limit into SL(2,R); hence, we cannot take the formal limit $\mathrm{D} \rightarrow \infty$ in equation (10) to examine the continuous scenario. We will base the continuous representation of linear canonical transformations in $\operatorname{SL}(2, \mathrm{R})$ in the formal sense on

$$
\mathrm{G}_{\infty}{ }^{\dagger} \mathrm{S}^{\wedge}{ }_{\alpha \mathrm{E}} \mathrm{G}_{\infty}=\mathrm{S}^{\wedge}{ }_{\alpha \mathrm{E} 0}
$$


4118

where $\alpha \mathrm{E}=\left(\alpha_{1}, \alpha_{2}\right) \in \mathrm{R} \times \mathrm{R}$ is a continuous phase space vector, $\hat{S}_{\vec{\alpha}}$ are elements of the continuous Schwinger operator basis [16] and $\alpha \mathrm{E}^{0}=\mathrm{R}: \alpha \mathrm{E}$ with $\mathrm{R} \in \mathrm{SL}(2, \mathrm{R})$ indicate the transformation matrix with real elements. From here on we will be confined to the continuous scenario in which we can drop the subscript $\infty$ from the canonical transformation generators $\hat{\mathcal{G}}_{\infty}$. The three one-parameter subgroups $\mathrm{g}_{\mathrm{j}},(\mathrm{j}=1,2,3)$ of $\mathrm{SL}(2, \mathrm{R})$, as conventionally represented by the three $2 \times 2$ matrices, correspond to

$$
\begin{array}{r}
\Omega_{1}(\psi)=\left(\begin{array}{cc}
\cosh \psi / 2 & \sinh \psi / 2 \\
\sinh \psi / 2 & \cosh \psi / 2
\end{array}\right) \quad \Omega_{2}(\theta)=\left(\begin{array}{cc}
\cos \theta / 2 & \sin \theta / 2 \\
-\sin \theta / 2 & \cos \theta / 2
\end{array}\right) \\
\Omega_{3}(\varphi)=\left(\begin{array}{cc}
\mathrm{e}^{\varphi / 2} & 0 \\
0 & \mathrm{e}^{-\varphi / 2}
\end{array}\right) \\
\cdot{ }_{\mathrm{j}} \in \mathrm{g}_{\mathrm{j}},-\infty<\psi<\infty,-\pi<\theta<\pi \text { and }-\pi 6 \phi 6 \pi \text {. A generic group element }
\end{array}
$$

$\mathrm{g} \in \mathrm{SL}(2, \mathrm{R})$ can be parametrized as

$$
g=\left(\begin{array}{ll}
\alpha & \beta \\
\gamma & \delta
\end{array}\right) \quad \text { where } \quad \operatorname{det} g=1
$$

with $\alpha, \beta, \gamma, \delta \in \mathrm{R}$ being functions of $\psi, \theta, \phi$.

The three Hermitian operators $K_{\hat{j}},(j=1,2,3)$ corresponding to the infinitesimal generators of the transformation in each subgroup respect the commutation relations

$$
\left[\mathrm{K}^{\wedge}{ }_{1}, \mathrm{~K}^{\wedge}\right]=\mathrm{i} \mathrm{K}^{\wedge} 3 \quad\left[\mathrm{~K}^{\wedge}{ }_{2}, \mathrm{~K}^{\wedge} 3\right]=\mathrm{iK}^{\wedge} 1 \quad\left[\mathrm{~K}^{\wedge}{ }_{1}, \mathrm{~K}^{\wedge} 3\right]=\mathrm{i} \mathrm{K}^{\wedge} 2 .
$$

We are particularly interested in the continuous irreducible representation of $\mathrm{K}^{\wedge} \mathrm{j}$, $(\mathrm{j}=1,2,3)$ in the canonical phase space parametrized by $\alpha \mathrm{E}$ in equation (18). This particular representation of the generators can be given by

$$
\begin{aligned}
& \mathrm{K}^{\wedge}{ }_{1}=-\mathrm{i}\left(\alpha_{1} \partial_{\alpha 2}+\alpha_{2} \partial_{\alpha 1}\right) / 2 \\
& \mathrm{~K}^{\wedge}{ }_{2}=-\mathrm{i}\left(\alpha_{1} \partial_{\alpha 2}-\alpha_{2} \partial_{\alpha 1}\right) / 2 \\
& \mathrm{~K}^{\wedge}{ }^{3}=-\mathrm{i}\left(\alpha_{1} \partial_{\alpha 1}-\alpha_{2} \partial_{\alpha 2}\right) / 2
\end{aligned}
$$

where each irreducible representation acts on the Hilbert space of homogeneous polynomials of degree 2 , and a definite parity where $2^{`} \in \mathrm{Z}$ with $\epsilon= \pm$ describing the odd $(-)$ and even(+) parity. Hence we characterize those irreducible representations using the standard notation by $\mathrm{T}_{\chi}\left(\mathrm{g}_{\mathrm{j}}\right)$ where $\chi=\left({ }^{\prime},\right)$ and the sector of the Hilbert space they belong to by $\mathrm{H}_{\chi}$. Also, in the family $\mathrm{T}_{\chi}\left(\mathrm{g}_{\mathrm{j}}\right)$ we are particularly interested in the diagonal representations of each generator in equation (22). 
2.2.1. Diagonal representations of the $\mathrm{j}$ th subgroup $\mathrm{g}_{\mathrm{j}}$. We now describe the eigenvectors $\mid e_{j}{ }^{x}\left(\gamma_{j}\right) i$ in the diagonal representations $T_{\chi}\left(g_{j}\right)$ characterized by a particular $j$ where $j=(1,2,3)$ with their corresponding projections on the canonical phase space $\left\langle\vec{\alpha} \mid e_{j}^{\chi}\left(\gamma_{j}\right)\right\rangle \equiv$ $e_{j}^{\chi}\left(\vec{\alpha}, \gamma_{j}\right)$. Considering the simplest case of ${ }^{`}=0$ first, $\left\langle\vec{\alpha} \mid e_{j}^{\chi}\left(\gamma_{j}\right)\right\rangle_{\text {are given by }}$

$$
\begin{aligned}
& T_{\chi}\left(\Omega_{1}\right): e_{1}^{\chi}\left(\vec{\alpha}, \gamma_{1}\right)=C_{1}\left(\frac{\alpha_{1}+\alpha_{2}}{\alpha_{1}-\alpha_{2}}\right)^{\mathrm{i} \gamma_{1}} \\
& T_{\chi}\left(\Omega_{2}\right): e_{2}^{\chi}\left(\vec{\alpha}, \gamma_{2}\right)=C_{2}\left(\frac{\alpha_{1}+i \alpha_{2}}{\alpha_{1}-i \alpha_{2}}\right)^{\gamma_{2}} \\
& T_{\chi}\left(\Omega_{3}\right): e_{3}^{\chi}\left(\vec{\alpha}, \gamma_{3}\right)=C_{3}\left(\frac{\alpha_{1}}{\alpha_{2}}\right)^{\mathrm{i} \gamma_{3}}
\end{aligned}
$$

where $\gamma_{\mathrm{j}} \in \mathrm{R}$ and $\mathrm{C}_{\mathrm{j}}$ are constants based on an appropriate normalization by the inner product $\left\langle e_{j}^{\chi}\left(\gamma_{j}\right) \mid e_{j}^{\chi}\left(\gamma_{j}^{\prime}\right)\right\rangle=\delta\left(\gamma_{j}-\gamma_{j}^{\prime}\right)$. Equations (23) imply that

$$
\hat{K}_{j}\left|e_{j}^{\chi}\left(\gamma_{j}\right)\right\rangle=\gamma_{j}\left|e_{j}^{\chi}\left(\gamma_{j}\right)\right\rangle \text {. }
$$

Within each subgroup $\mathrm{g}_{\mathrm{j}},(\mathrm{j}=1,2,3)$ we now define the unitary subgroup elements $\hat{\mathcal{G}}_{j} \in g_{j}$ such that

$$
\hat{\mathcal{G}}_{j}^{\Gamma_{j}}=\mathrm{e}^{-\mathrm{i} \Gamma_{j} \hat{K}_{j}}
$$

where $0_{j} \in R,(j=1,2,3)$. The representations of equations (25) in terms of $2 \times 2$ matrices in the phase space $\vec{\alpha}=\left(\begin{array}{c}\alpha_{1} \\ \alpha_{2}\end{array}\right)$ are given by the $\cdot{ }_{j}$ in equation (19), namely, the action of the each group element $\mathrm{G}^{\wedge} \mathrm{j}$ in equation (18) is given by

$$
\left(\hat{\mathcal{G}}_{j}^{\Gamma_{j}}\right)^{\dagger} \hat{S}_{\vec{\alpha}} \hat{\mathcal{G}}_{j}^{\Gamma_{j}} \equiv \hat{S}_{\alpha^{\prime}}=\hat{S}_{\Omega_{j}: \vec{\alpha}} .
$$

Within each subgroup $\mathrm{g}_{\mathrm{j}}$ there exists, in the Schwinger sense [15], a special canonical partner denoted by $\hat{\mathcal{O}}_{j}$ of $\hat{\mathcal{G}}_{j}$ such that

$$
\hat{\mathcal{G}}_{j}^{\Gamma_{j}} \hat{\mathcal{O}}_{j}^{\zeta_{j}}=\mathrm{e}^{-\mathrm{i} \Gamma_{j} \zeta_{j}} \hat{\mathcal{O}}_{j}^{\zeta_{j}} \hat{\mathcal{G}}_{j}^{\Gamma_{j}} .
$$

Equation (27) implies that

$$
\hat{\mathcal{G}}_{j}^{\Gamma_{j}}\left|e_{j}^{\chi}\left(\gamma_{j}\right)\right\rangle=\mathrm{e}^{-\mathrm{i} \Gamma_{j} \gamma_{j}}\left|e_{j}^{\chi}\left(\gamma_{j}\right)\right\rangle \quad \hat{\mathcal{O}}_{j}^{\zeta_{j}}\left|e_{j}^{\chi}\left(\gamma_{j}\right)\right\rangle \sim\left|e_{j}^{\chi}\left(\gamma_{j}+\zeta_{j}\right)\right\rangle
$$

where the $\sim$ sign indicates that the equality holds up to an indeterminable phase factor which we consider to be irrelevant. Then equation (28) provides a representation for the operator $\hat{\mathcal{O}}_{j}$ in the diagonal representation of $\hat{\mathcal{G}}_{j}$. To complete the picture we consider, for each subgroup $\mathrm{g}_{\mathrm{j}}$, the diagonal representation of the $\hat{\mathcal{O}}_{j}$ operator such that

$$
\hat{\mathcal{O}}_{j}^{\zeta_{j}}\left|f_{j}^{\chi}\left(\eta_{j}\right)\right\rangle=\mathrm{e}^{\mathrm{i} \zeta_{j} \eta_{j}}\left|f_{j}^{\chi}\left(\eta_{j}\right)\right\rangle
$$

From equations (27) the action of $\mathcal{G}^{\hat{\Gamma}_{j}}$ on this basis can be found as

$$
\hat{\mathcal{G}}_{j}^{\Gamma_{j}}\left|f_{j}^{\chi}\left(\eta_{j}\right)\right\rangle \sim\left|f_{j}^{\chi}\left(\eta_{j}+\Gamma_{j}\right)\right\rangle \text {. }
$$

The $\sim$ again indicates that there is an overall indeterminable phase which we ignore without any loss of generality. Then equations (27)-(30) completely determine, in the weak sense, the properties of the operator $\operatorname{pair}(\hat{\mathcal{G}}, \hat{\mathcal{O}})$ within the Hilbert space of each subgroup $\mathrm{g}_{\mathrm{j}}$ as three 
different realizations of the Schwinger operator basis. The connection between the eigenbasis $\left|e_{j}^{\chi}\left(\gamma_{j}\right)\right\rangle$ and $\left|f_{j}^{\chi}\left(\eta_{j}\right)\right\rangle_{\text {is given by the Fourier transformation }}$

$\mid f_{j} \chi\left(\eta_{j}\right) i=Z d \gamma_{j}$ e-i $\gamma_{j} \eta_{j}\left|e_{j} x\left(\gamma_{j}\right) i \quad\right| e j x\left(\gamma_{j}\right) i=Z d \eta_{j} e_{i \eta \gamma_{j}} \mid f_{j}\left(\eta_{j}\right) i$

where in short notation $\left|f_{j}^{\chi}\left(\eta_{j}\right)\right\rangle=\hat{\mathcal{F}}\left|e_{j}^{\chi}\left(\gamma_{j}\right)\right\rangle$ with $(\hat{\mathcal{F}})_{\eta_{j}, \gamma_{j}}=\left(\left\langle f_{j}^{\chi}\left(\eta_{j}\right) \mid e_{j}^{\chi}\left(\gamma_{j}\right)\right\rangle\right)$ describing the matrix elements parametrized by $\left(\eta_{\mathrm{j}}, \gamma_{\mathrm{j}}\right)$ of the unitary Fourier operator $\mathrm{F}^{\wedge}$. Via equation (31) a Fourier automorphism is implied between the two eigenspaces for each $\mathrm{j}$ as

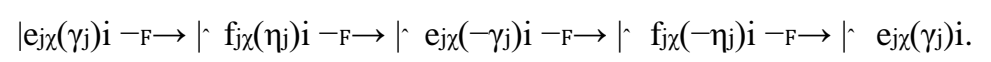

2.2.2. Diagonal representations of the entire group g. We now shift our attention from the parametrization of the diagonal representations of $g_{j}$ to those of the entire group $g$ of which the three-parameter group element will be denoted by $\mathrm{G}^{\wedge}$. For convenience of the calculations we adopt the unitary canonical form for $\mathrm{G}^{\wedge}$ as [27]

$$
\mathrm{G}^{\wedge} 3=\mathrm{e}-\mathrm{i} 3 \mathrm{E} \cdot \mathrm{KE}^{\wedge} \quad 3 \mathrm{E}=(31,32,33) \quad \overrightarrow{\hat{K}}=\left(\hat{K}_{1}, \hat{K}_{2}, \hat{K}_{3}\right)
$$

where $3 \mathrm{E}$ is defined on the $\mathrm{SL}(2, \mathrm{R})$ invariant group manifold characterized by the invariant $3^{2}=\Lambda_{1}^{2}+\Lambda_{3}^{2}-\Lambda_{2}^{2} . \quad$ Adopting the particular parametrization $\Lambda_{1}=\Lambda \sin a \cosh b$, $3_{2}=3 \sin$ a sinh b, and $3_{3}=3 \operatorname{cosa}$, equation (33) can be obtained, for example, from $\mathcal{G}_{3}^{\Lambda}$ in equation (25) by the unitary transformation

$$
\hat{\mathcal{G}}^{\Lambda}=\left(\hat{\mathcal{T}}_{23}^{(-a, b)}\right)^{\dagger} \hat{\mathcal{G}}_{3}^{\Lambda} \hat{\mathcal{T}}_{23}^{(-a, b)} \quad \text { where } \quad \hat{\mathcal{T}}_{23}^{(-a, b)}=\hat{\mathcal{G}}_{2}^{-a} \hat{\mathcal{G}}_{3}^{b} .
$$

Since $\mathrm{G}^{\wedge}{ }^{0}$ in equation (33) is an element of $\operatorname{SL}(2, \mathrm{R})$, the irreducible representations also act on the homogeneous polynomials of degree 2 and parity $\epsilon= \pm$ in $H_{\chi}$.

(i) Continuous diagonal representations.

Similar to equations (24) and to the first set in equations (28) for the subgroups, we now seek the eigenvectors $\mid h^{x}(\gamma) i \in \mathrm{H}_{\chi}$ of $\mathrm{G}^{\wedge 3}$ such that

$$
\mathrm{G}^{\wedge} 3\left|\mathrm{~h}_{\chi}(\gamma) \mathrm{i}=\mathrm{eie}_{\mathrm{i}}\right| \mathrm{h}_{\chi}(\gamma) \mathrm{i} \quad \mathrm{A} \in \mathrm{R}
$$

where $\mathrm{A}$ and $\mid \mathrm{h}^{x}(\gamma)$ i are to be found from the eigenproblem in equation (35). Equation (34) suggests that

$$
\left|h^{\chi}(\gamma)\right\rangle=\left(\hat{\mathcal{T}}_{23}^{(-a, b)}\right)^{\dagger}\left|e_{3}^{\chi}(\gamma)\right\rangle
$$

where $A=-3$ in equation (35). Hence $\mid h^{x}(\gamma)$ i spans the eigenspace of the unitary operator $\chi$ in equation (33) with3, $\gamma \in \mathrm{R}$. The orthonormality of the eigenbasis $\mid \mathrm{h}(\gamma) \mathrm{i}$ is guaranteed by the unitary transformation in equation (36) and the orthonormality of the eigenbasis $\left|e_{3}^{\chi}(\gamma)\right\rangle$. A 
phase space representation for $\mid \mathrm{h}^{x}(\gamma)$ i similar to equations (23) can be found by projecting it on the phase space vector $\alpha \mathrm{E}$ as

$$
\left\langle\vec{\alpha} \mid h^{\chi}(\gamma)\right\rangle \equiv h^{\chi}(\vec{\alpha}, \gamma)=\left\langle\vec{\alpha}\left|\left(\hat{\mathcal{T}}_{23}^{(-a, b)}\right)^{\dagger}\right| e_{3}^{\chi}(\gamma)\right\rangle \quad \hat{\mathcal{T}}_{23}^{(-a, b)}\left(\begin{array}{c}
\alpha_{1} \\
\alpha_{2}
\end{array}\right)=\Omega_{3}(b) \Omega_{2}(a)\left(\begin{array}{c}
\alpha_{1} \\
\alpha_{2}
\end{array}\right)
$$

where ${ }_{2}$ (a) and $\bullet{ }_{3}$ (b) are implied by equation (19). The unitary-canonical partner of $\hat{G}$ can be found similarly as it was done for the subgroups. Defining 0^ such that

$$
\mathrm{G}^{\wedge} 3 \mathrm{O}^{\wedge} \zeta=\mathrm{e}-\mathrm{i} 3 \zeta \mathrm{O}^{\wedge} \zeta \mathrm{G}^{\wedge} 3
$$

we find

$$
\begin{array}{lrl}
\hat{\mathcal{G}}^{\Lambda}\left|h^{\chi}(\gamma)\right\rangle & =\mathrm{e}^{-\mathrm{i} \Lambda \gamma}\left|h^{\chi}(\gamma)\right\rangle & \hat{\mathcal{O}}^{\zeta}\left|h^{\chi}(\gamma)\right\rangle \sim\left|h^{\chi}(\gamma+\zeta)\right\rangle \\
\hat{\mathcal{O}}^{\zeta}\left|k^{\chi}(\eta)\right\rangle=\mathrm{e}^{\mathrm{i} \zeta \eta}\left|k^{\chi}(\eta)\right\rangle & \hat{\mathcal{G}}^{\Lambda}\left|k^{\chi}(\eta)\right\rangle \sim\left|k^{\chi}(\eta+\Lambda)\right\rangle
\end{array}
$$

where we will again neglect the overall phases in the second column of the relations above. The basis vectors in equation (39) are connected by the Fourier transformation

$$
\left|\mathrm{k}^{x}(\eta)^{\mathrm{i}=} \mathrm{Z} \mathrm{d} \gamma \mathrm{e}^{-\mathrm{i} \gamma \eta}\right| \mathrm{h}^{x}(\gamma) \mathrm{i} \quad \quad\left|\mathrm{h}^{x}(\gamma)^{\mathrm{i}=} \mathrm{Zd \eta} \mathrm{e}^{\mathrm{i} \gamma \eta}\right| \mathrm{k}^{x}(\eta) \mathrm{i}
$$

and a similar automorphism to equation (32) between $\mid h^{x}(\gamma) i$ and $\mid k^{x}(\eta)$ i as well as to equation (5) at the operator level between $\mathrm{G}^{\wedge}$ and $\mathrm{O}^{\wedge}$ can be written.

(ii) Discrete diagonal representations.

In examining the discrete representations of the entire group we start with the diagonal ones $\left|e_{2}^{\chi}(m)\right\rangle$ of $\hat{K}_{2}$ and associate with them the eigenfunctions

$$
\left\langle\vec{\alpha} \mid e_{2}^{\chi}(m)\right\rangle \equiv e_{2}^{\chi}(\vec{\alpha} ; m)=N_{2}^{(\ell)}\left(\alpha_{1}^{2}+\alpha_{2}^{2}\right)^{\ell}\left(\frac{\alpha_{1}+\mathrm{i} \alpha_{2}}{\alpha_{1}-\mathrm{i} \alpha_{2}}\right)^{m}
$$

where $\left.\mathrm{N}_{2}{ }^{(}\right)$is a normalization based on an inner product $\left\langle e_{2}^{\chi}(\vec{\alpha} ; m) \mid e_{2}^{\chi}\left(\vec{\alpha} ; m^{\prime}\right)\right\rangle=\delta_{m, m^{\prime}}$. For the state in equation (41) we have

$$
\begin{aligned}
& \hat{K}_{2}\left|e_{2}^{\chi}(m)\right\rangle=m\left|e_{2}^{\chi}(m)\right\rangle \\
& \hat{K}_{+}\left|e_{2}^{\chi}(m)\right\rangle=-(\ell-m)\left|e_{2}^{\chi}(m+1)\right\rangle \\
& \hat{K}_{-}\left|e_{2}^{\chi}(m)\right\rangle=(\ell+m)\left|e_{2}^{\chi}(m-1)\right\rangle
\end{aligned}
$$

where $\hat{K}_{ \pm}=\left(\hat{K}_{1} \pm \mathrm{i} \hat{K}_{3}\right)$ and the Casimir element $\hat{K}^{2}=1 / 2\left(\hat{K}_{+} \hat{K}_{-}+\hat{K}_{-} \hat{K}_{+}\right)-K_{2}^{2}$ has eigenvalue $`(+1)$. In terms of the $` 6=0$ representation in equations $(41)$ and $(42)$, the continuous representations that we used in equation (23) correspond to the $`=0$ case. A general eigenvector for a group element in equation (33) can be found similarly as in the continuous case by redefining $3^{\mathrm{E}}=\left(3_{1}, 3_{2}, 3_{3}\right)$ as, for instance, $3_{1}=3 \sinh \mathrm{a}^{0} \sin \mathrm{b}^{0}, 3_{2}=3 \cosh$ $a^{0}, 3_{3}=3 \sinh a^{0} \cos b^{0}$ and the transformation

$$
\hat{\mathcal{G}}^{\Lambda}=\left(\hat{\mathcal{T}}_{12}^{\left(a^{\prime}, b^{\prime}\right)}\right)^{\dagger} \hat{\mathcal{G}}_{2}^{\Lambda} \hat{\mathcal{T}}_{12}^{\left(a^{\prime}, b^{\prime}\right)} \quad \text { where } \quad \hat{\mathcal{T}}_{12}^{\left(a^{\prime}, b^{\prime}\right)}=\hat{\mathcal{G}}_{1}^{a} \hat{\mathcal{G}}_{2}^{b}
$$


The eigenvectors $\mid h^{\chi}(m)$ i of a general group element $G^{\wedge}$ are then associated with the functions

in $\mathrm{H}_{\chi}$

$\left\langle\vec{\alpha} \mid h^{\chi}(m)\right\rangle \equiv h^{\chi}(\vec{\alpha} ; m)=\left\langle\vec{\alpha}\left|\left(\hat{\mathcal{T}}_{12}^{\left(a^{\prime}, b^{\prime}\right)}\right)^{\dagger}\right| e_{2}^{\chi}(m)\right\rangle \quad \hat{\mathcal{T}}_{12}^{\left(a^{\prime}, b^{\prime}\right)}\left(\begin{array}{l}\alpha_{1} \\ \alpha_{2}\end{array}\right)=\Omega_{2}\left(-b^{\prime}\right) \Omega_{1}\left(a^{\prime}\right)\left(\begin{array}{l}\alpha_{1} \\ \alpha_{2}\end{array}\right)$

where ${ }_{2}(-b)$ and $\bullet{ }_{1}$ (a) are implied by equations (19). To establish the representation of the unitary canonical pair $\left(\mathrm{G}^{\wedge}, \mathrm{O}^{\wedge}\right)$ in the discrete case we consider

$$
\mathrm{G}^{\wedge}{ }_{30} \hat{\mathrm{r}}=\mathrm{e}-\mathrm{i} 3 \mathrm{rO}^{\wedge} \mathrm{rG}^{\wedge} 3
$$

corresponding to a change $\zeta \rightarrow \mathrm{r} \in \mathrm{Z}$ in equation (38). For the system of eigenvectors we

find

$$
\begin{aligned}
& \hat{\mathcal{G}}^{\Lambda}\left|h^{\chi}(m)\right\rangle=\mathrm{e}^{-\mathrm{i} \Lambda m}\left|h^{\chi}(m)\right\rangle \quad \hat{\mathcal{O}}^{r}\left|h^{\chi}(m)\right\rangle \sim\left|h^{\chi}(m+r)\right\rangle \\
& \hat{\mathcal{O}}^{r}\left|k^{\chi}(\eta)\right\rangle=\mathrm{e}^{\mathrm{i} \eta r}\left|k^{\chi}(j, \eta)\right\rangle \quad \hat{\mathcal{G}}^{\Lambda}\left|k^{\chi}(\eta)\right\rangle \sim\left|k^{\chi}(\eta+\Lambda)\right\rangle
\end{aligned}
$$

where $3, \eta \in R$. The eigenspaces are connected by the (discrete and continuous) Fourier transformation as

$$
k^{\chi}(\vec{\alpha} ; \eta)=\sum_{m} \mathrm{e}^{-\mathrm{i} \eta m} h^{\chi}(\vec{\alpha} ; m) \quad h^{\chi}(\vec{\alpha} ; m)=\int \mathrm{d} \eta \mathrm{e}^{\mathrm{i} m \eta} k^{\chi}(\vec{\alpha} ; \eta) .
$$

The equations (46) and (47) conclude our brief treatment of the unitary continuous phase space representations of the canonical $\mathrm{G}^{\wedge}, \mathrm{O}^{\wedge}$ pair in the weak matrix element sense.

\section{Implications for the Hamiltonian systems with dynamical symmetry group and generalized AA operators}

Recently, an algebraic approach was studied by Wang and Chu [28] in the solution of the onedimensional inverse problem for the Hamiltonian systems with dynamical group symmetry. In the simplest case of one dimension, the inverse problem reduces to that of finding an operator regarding the dynamical symmetry group of the system such that it will be invariant under either a certain subgroup or the entire group of canonical transformations. The group of canonical transformations then naturally reflects the properties of the dynamical symmetry of the system. For one-dimensional autonomous systems, the invariant operator under canonical transformations corresponds to the generalized action operator, by which the Hamiltonian of the entire system can be fully described. In the following, we will examine the quantum canonical transformation group as the dynamical symmetry group for an Hamiltonian system and derive the equations of motion describing the time evolution of the generalized quantum AA operators.

LetusassumethattheHamiltonianH^ ${ }^{\wedge}$ describingthedynamicsofasystemwithonedegree of freedom is represented by

$$
\mathrm{H}^{\wedge} \equiv \mathrm{H}\left(\mathrm{K}^{\wedge} 1, \mathrm{~K}^{\wedge} 2, \mathrm{~K}^{\wedge} 3\right)
$$


where $\hat{K}_{j}$ are the Hermitian generators of the $\mathrm{sl}(2, \mathrm{R})$ canonical transformation algebra in equation (33). The simplest but a sufficiently general example of an Hamiltonian with a dynamical group symmetry can then be obtained if the Hamiltonian is a real function of the linear superposition of $\hat{K}_{j}$ as,

$$
\hat{\mathcal{H}}=\mathcal{H}\left(P \hat{K}_{1}+Q \hat{K}_{2}+R \hat{K}_{3}\right) \quad P, Q, R \in \mathbb{R} .
$$

Here, we do not make any assumptions aside from the one that $\mathrm{H}$ is a real valued and a well behaved function. Our focus will be on those unitary elements of the dynamical symmetry group that can be described by

$$
\hat{\mathcal{G}}^{\Gamma}=\mathrm{e}^{-\mathrm{i} \Gamma\left(P \hat{K}_{1}+Q \hat{K}_{2}+R \hat{K}_{3}\right)}
$$

where the Hamiltonian in equation (49) becomes

$$
\hat{\mathcal{H}}=\left.\mathcal{H}\left(\mathrm{i} \frac{\partial}{\partial \Gamma}\right) \hat{\mathcal{G}}^{\Gamma}\right|_{\Gamma=0} .
$$

The time-dependent eigenvectors $\mid \psi_{\gamma}{ }^{\gamma}(\mathrm{t}) \mathrm{i}$ of $\mathrm{H}^{\wedge}$ and the eigenenergies in the continuous representation are given by

$$
\left\langle\vec{\alpha} \mid \psi_{\gamma}^{\chi}(t)\right\rangle=\mathrm{e}^{\mathrm{i} E_{\gamma} t} h^{\chi}(\vec{\alpha}, \gamma) \quad E_{\gamma}=\mathcal{H}(\gamma)
$$

where $\mathrm{h}^{\chi}(\alpha, \gamma) \mathrm{E}$ is given by equation (37). Here, the unitary canonical partner 0 to $\mathrm{G}$ plays the role of the unitary angle operator and is defined by equation (38). The time evolution of

$0 ` \zeta$ is given by $\mathrm{d}$

$$
\left\langle\psi_{\gamma^{\prime}}\left|\hat{\mathcal{O}}^{\zeta}\right| \psi_{\gamma}\right\rangle=\left\langle\psi_{\gamma^{\prime}}\left|\left[\hat{\mathcal{H}}, \hat{\mathcal{O}}^{\zeta}\right]\right| \psi_{\gamma^{\prime}}\right\rangle=-\left\langle\psi_{\gamma^{\prime}}\left|\hat{\mathcal{O}}^{\zeta}\right| \psi_{\gamma}\right\rangle\{\mathcal{H}(\gamma+\zeta)-\mathcal{H}(\gamma)\} \quad \text { i }
$$

If we symbolically associate an Hermitian phase operator at $\zeta=0$ with

equation (53) becomes, in

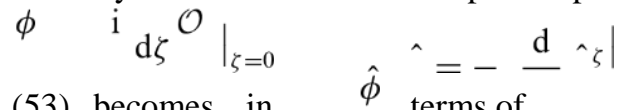

$$
\lim _{\zeta \rightarrow 0}\left\langle\psi_{\gamma-\zeta}\left|\frac{\mathrm{d}}{\mathrm{d} t} \hat{\phi}\right| \psi_{\gamma}\right\rangle=-\frac{\mathrm{d} E_{\gamma}}{\mathrm{d} \gamma} \lim _{\zeta \rightarrow 0}\left\langle\psi_{\gamma-\zeta} \mid \psi_{\gamma}\right\rangle_{\mid}
$$

which is the quantum analogue of the classical equation of motion for the canonical angle variable. Hence the unitary operator should be considered as the quantum angle operator which is the unitary canonical partner of the action operator $\mathrm{G}^{\wedge}$.

The properties of the dynamical group symmetry can also be examined by using the discrete representation. In this case, equation (51) is still valid whereas (52) becomes

$$
\left\langle\vec{\alpha} \mid \psi_{m}^{\chi}(t)\right\rangle=\mathrm{e}^{\mathrm{i} E_{m} t} h^{\chi}(\vec{\alpha} ; m) \quad E_{m}=\mathcal{H}_{(\mathrm{m})}
$$

where $\left\langle\vec{\alpha} \mid \psi_{m}^{\chi}(t)\right\rangle$ is the time-dependent eigenvector, $\mathrm{h}^{\chi}(\alpha \mathrm{E} ; \mathrm{m})$ is given by equation (44) and $E_{m}=H(m)$ is the discrete eigenenergy spectrum depending on the discrete eigenstate index $r$ $\mathrm{m}$. The appropriate angle operator $\hat{\mathrm{O}}_{\mathrm{r}}$ in the discrete case has been studied in equations (45) 
and (46). The nature of the operator $\mathrm{O}^{\wedge}$ becomes more transparent if we examine the derivative of equation (45) with respect to 3 at $3=0$. This can be readily evaluated as

$$
\left.\mathrm{d} \quad \rightarrow(45)\right|_{\Lambda=0} \Longrightarrow\left[\vec{n} \cdot \overrightarrow{\hat{K}}, \hat{\mathcal{O}}^{r}\right]=-r \hat{\mathcal{O}}^{r}
$$
equation (57) $\mathrm{d} 3$

whichisthegeneralizedSusskind-Glogower-Carruthers-Nietocommutationrelation [5,7,15] for the generalized radial number operator $\vec{n} \cdot \overrightarrow{\hat{K}}$ and the generalized unitary phase operator $\mathrm{O}^{\wedge}$. The time evolution of $\mathrm{O}^{\wedge}$ is then

d

$\begin{array}{llllllllllll}\mathrm{d} & \chi & \mathrm{r} & \chi & \chi & \mathrm{r} & \chi & & \chi & \mathrm{r} & \chi\end{array}$

$\mathrm{i} \_h \psi_{\mathrm{mo}}\left|\mathrm{O}^{\wedge}\right| \psi \mathrm{mi}=\mathrm{h} \psi \mathrm{mo}\left|\left[\mathrm{H}^{\wedge}, \mathrm{O}^{\wedge}\right]\right| \psi \mathrm{mi}=-\mathrm{h} \psi \mathrm{mo} 0 \mathrm{O}^{\wedge} \mid \psi \mathrm{mi}\{\mathrm{H}(\mathrm{m}+\mathrm{r})-\mathrm{H}(\mathrm{m})\}$

which is the equation of motion for the unitary canonical angle operator of the generalized oscillator with a discrete spectrum $E_{m}=H(m)$. Here, two results are in order. The first is that we have found a correspondence between the classical and quantum AA formalisms for Hamiltonian systems with a dynamical group symmetry of the type given by equations (49) and (50). The second result is the equivalence of the quantum action operator to the generators of canonical transformations as well as that of the unitary-canonical angle operator to the unitary-canonical phase operator.

Although the continuous and discrete representations of the AA operators are similar, depending on the continuous/discrete nature of the eigenenergy spectrum, one or the other is more convenient in the formulation of a physical problem. This will be more transparent in the next section when we discuss the AA formalism of a generalized oscillator.

\subsection{Implications for the generalized oscillator Hamiltonian and the quantum phase operator}

Inthisandthefollowingsectionswerefertotheappendixwhichincludessomerelevantpartsof [15]. There, it is shown that the QHO algebra is recovered in the infinite-dimensional limit (i.e. D $\rightarrow \infty$ hence $\mathrm{q} \rightarrow 1$ ) of the admissible q-oscillator algebraic realization in equations (A.4).

The importance of the naturally emerging admissible q-oscillator realizations is that they admit an algebraic formulation of the quantum phase problem and also provide a natural basis to examine the harmonic oscillator phase in the infinite-dimensional limit of the algebra in equations (A.4). Respecting the historical development, we will nevertheless start with a brief outline of the phase problem using the dynamical continuous symmetry group of the QHO. The generators $\hat{K}_{i},(i=1,2,3)$ of the dynamical $\mathrm{sl}(2, \mathrm{R})$ symmetry of the $\mathrm{QHO}$ in the $\mathrm{x}, \hat{\mathrm{p}}$ ^ representation are given by

$$
\hat{K}_{1}=\frac{1}{4}\left(\hat{x}^{2}-\hat{p}^{2}\right) \quad \hat{K}_{2}=\frac{1}{4}\left(\hat{x}^{2}+\hat{p}^{2}\right) \quad \hat{K}_{3}=\frac{1}{4}(\hat{x} \hat{p}+\hat{p} \hat{x}) \quad\left[\mathrm{x}, \hat{p} \mathrm{p}^{\wedge}\right]=\mathrm{i}
$$

where the generators respect equations (21). Our first attempt will be to find the unitary canonical partner to $\mathrm{K}^{\wedge} 3$ in equations (59). With $\mathrm{p}^{\wedge} \rightarrow-\mathrm{i} \partial / \partial \mathrm{x}$, the eigenproblem for $\hat{K}_{3}$ yields

$$
\mathrm{K}^{\wedge} 3\left|\psi_{3}\left(\gamma_{3}\right) \mathrm{i}=\gamma_{3}\right| \psi_{3}\left(\gamma_{3}\right) \mathrm{i} \gamma_{3} \in \mathrm{R} \mathrm{hx} \mid \psi_{3}\left(\gamma_{3}\right) \mathrm{i}=\mathrm{N}_{3} \mathrm{X}_{\left(2 \gamma_{3}-1 / 2\right)}(60) \text { where } \mathrm{N}_{3} \text { is a }
$$

normalization based on an inner product. Hence, for $\hat{\mathcal{G}}_{3}^{\Gamma_{3}}=\mathrm{e}^{-\mathrm{i} \Gamma_{3} \hat{K}_{3}}$, 
The unitary canonical partner $\hat{\mathcal{O}}_{3}$ to $\hat{\mathcal{G}}_{3}$, such that equation (27) is satisfied for $\mathrm{i}=3$, can be found following the steps leading to equations (28)-(30). The $\hat{\mathcal{O}}_{30 \text { operator for an arbitrary and }}^{\zeta}$ real $\zeta$ is given by

$$
\hat{\mathcal{O}}_{3}^{\zeta}=\int_{-\infty}^{\infty} \mathrm{d} \gamma_{3}\left|\psi\left(\gamma_{3}+\zeta\right)\right\rangle\left\langle\psi\left(\gamma_{3}\right)\right|
$$

or equivalently by

$$
\hat{\mathcal{O}}_{3}^{\zeta}=\int_{-\infty}^{\infty} \mathrm{d} \eta_{3} \mathrm{e}^{\mathrm{i} \zeta \eta_{3}}\left|\tilde{\psi}\left(\eta_{3}\right)\right\rangle\left\langle\tilde{\psi}\left(\eta_{3}\right)\right|
$$

where

$$
\left|\tilde{\psi}\left(\eta_{3}\right)\right\rangle=\int_{-\infty}^{\infty} \mathrm{d} \gamma_{3} \mathrm{e}^{-\mathrm{i} \eta_{3} \gamma_{3}}\left|\psi\left(\gamma_{3}\right)\right\rangle
$$

namely, the existence of $\hat{\mathrm{O}}_{3}$ is manifested by the presence of a complete spectrum of $\mathrm{K}^{\wedge} 3$ on the real axis and, in return, $\mathrm{O}^{\wedge} 3$ and $\mathrm{G}^{\wedge} 3$ are connected by a Fourier automorphism. A similar procedure can also be applied to $\mathrm{K}^{\wedge} 1$ in equation (59) since the eigenspectrum of this operator also spans the symmetric positive and negative values on the entire real axis. However, there is a problem with the $\mathrm{K}_{2} 2$ operator. Because of the fact that $\hat{K}_{2}$ in equations (59) is a nonnegative operator, its eigenspectrum spans only the positive real axis. Hence, the Fourier automorphism is not applicable to $\mathrm{K}^{\wedge} 2$ and, in return, the unitary canonical partner to $\hat{\mathcal{G}}_{2}$ cannot be found. This problem was attacked from a completely different perspective a long time ago in the elegant work of Boyer and Wolf [11] where they made use of the unitary isomorphism between the radial representation of the dynamical $\mathrm{SL}(2, \mathrm{R})$ symmetry group of the multidimensional QHO with an added centrifugal term of arbitrary strength and the representation of the same group on the unit circle. Through this unitary mapping the space of square integrable functions on the unit circle is an inner product space endowed with a translationally invariant non-local measure. However, the drawback of this elegant method is that, the unitary irreducible representations are not single-valued under full rotations on the unit circle and this applies particularly to the standard one-dimensional QHO.

The phase problem in the $\mathrm{QHO}$ being the central theme of this paper, we suggest here and in the following sections an alternative and, perhaps, a formally simpler way of looking into the problem. We start with the admissible q-oscillator realization in equation (A.4) with the operators $\mathrm{A}^{\wedge} \mathrm{A}^{\wedge \uparrow}$ and $\mathrm{Q}^{\wedge}$ and approach the $\mathrm{QHO}$ algebra by extending $\mathrm{D}$ to infinity. We formally express the generalized oscillator Hamiltonian as (in analogy with equation (51))

$$
\mathrm{H}^{\wedge}=\mathrm{H}(\mathrm{N})^{\wedge}=\left.\mathrm{H}(\mathrm{q} \partial / \partial \mathrm{q}) \mathrm{Q}^{\wedge}\right|_{\mathrm{q} \rightarrow 1}
$$

where the limit $\mathrm{q} \rightarrow 1$ is achieved simultaneously with $\uparrow \mathrm{D} \rightarrow \infty$. The deformed algebra defined by the elements $\mathrm{A}^{\wedge} \mathrm{A}^{\wedge}$ and $\mathrm{Q}^{\wedge}$ is an admissible version of the well known (deformed) qoscillator algebra naturally admitting real and non-negative norms in the finite-dimensional cyclic Hilbert space ${ }^{\mathrm{D}} \mathrm{H}_{\mathrm{D}}$. Since the deformation parameter ${ }^{\dagger} \mathrm{q}=\mathrm{e}^{-\mathrm{i} \gamma_{0} \mathrm{mE} \times \mathrm{Em}_{0}}$ is a pure phase with 
the property that $\mathrm{q}=1$, the operators $\mathrm{A}, \mathrm{A}^{\wedge}, \mathrm{Q}^{\wedge}$ act on in the finite-D-dimensional cyclic Fock space spanned by the cyclic orthonormal vectors $\{\mid \mathrm{ni}\}=\{|\operatorname{ni06n6(D-1)} ;| \mathrm{ni}=\mid \mathrm{n}+\mathrm{Di}\}$

with

$$
\begin{aligned}
& A|n\rangle=\sqrt{f(n)}|n-1\rangle \\
& \hat{A}^{\dagger}|n\rangle=\sqrt{f(n+1)}|n+1\rangle \\
& \left.\hat{Q}|n\rangle=q^{n} n\right\rangle \quad q=\mathrm{e}^{-\mathrm{i} \gamma_{0} \vec{m} \times \vec{m}^{\prime}}
\end{aligned}
$$

with $06 \mathrm{f}(\mathrm{n})$ and $\mathrm{f}(\mathrm{n})=\mathrm{f}(\mathrm{n}+\mathrm{D})$ where

$$
f(n)=\frac{q^{n+(D-1) / 2}-q^{-n-(D-1) / 2}}{q-q^{-1}}+C \quad C=\frac{2}{\left|q-q^{-1}\right|} \neq 0 .
$$

The algebra in equation (A.4) and the relations (64) admit a unitary canonical partner to Q', i.e. the unitary quantum phase operator $\hat{\mathcal{E}}_{\phi}$

$$
\hat{\mathcal{E}}_{\phi}=\sum_{n=0}^{D-1}|n-1\rangle\langle n| \quad \hat{\mathcal{E}}_{\phi}^{D} \equiv \mathbb{I}
$$

such that

$$
\begin{gathered}
\left.\hat{\mathcal{E}}_{\phi} \text { are }\{|\phi\rangle\}=\{\phi\rangle_{0 \leqslant r \leqslant(D-1)} ;|\phi\rangle_{r+D} \equiv|\phi\rangle_{r}\right\} \in H_{D} \text { with }_{r^{\prime}}\langle\phi \mid \phi\rangle_{r}=\delta_{r^{\prime}, r} \\
\hat{Q}^{\Gamma} \hat{\mathcal{E}}_{\phi}^{\lambda}=q^{\Gamma \lambda} \hat{\mathcal{E}}_{\phi}^{\lambda} \hat{Q}^{\Gamma} \quad \underset{\Gamma, \lambda \in \mathbb{R}}{ } .
\end{gathered}
$$

The eigenvectors of

where

$$
\hat{\mathcal{E}}_{\phi}|\phi\rangle_{r}=\mathrm{e}^{\mathrm{i} \gamma_{0} r}|\phi\rangle_{r} \quad|\phi\rangle_{r}=\frac{1}{\sqrt{D}} \sum_{n=0}^{D-1} \mathrm{e}^{\mathrm{i} \gamma_{0} n r}|n\rangle \quad \gamma_{0}=\frac{2 \pi}{D} .
$$

The dynamical time evolution of $\mathrm{E}^{\wedge} \varphi$ for the generalized Hamiltonian in equation (63) is given by equation (58) as

$$
\begin{aligned}
& \begin{array}{lllllll}
t & \left.\right|_{\phi} \mid & -q & \left.\right|_{q \rightarrow 1} & \mathrm{~d} & \\
& & & & \mathrm{r} & \mathrm{n} \\
& & & & &
\end{array} \\
& \mathrm{n} \\
& \mathrm{i} \_\mathrm{hn}^{0} \mathrm{E}^{\wedge}\left|\mathrm{ni}=-\mathrm{hn}^{0}\right| \mathrm{E}^{\wedge} \mathrm{ni}\{\mathrm{H}(\mathrm{q} \partial / \partial \mathrm{q})(\mathrm{q}) \quad\} \mathrm{d} \\
& =-\operatorname{hno}\left|E_{\varphi}{ }^{\mathrm{r}}\right| \operatorname{ni}\{\mathrm{H}(\mathrm{n}+\mathrm{r})-\mathrm{H}(\mathrm{n})\}
\end{aligned}
$$

and a close inspection of equation (69) with (58) indicates that the pair $\left(\hat{Q}, \hat{\mathcal{E}}_{\phi}\right)$ is the corresponding unitary AA pair for the Hamiltonian in equation (63). As mentioned before, the $\mathrm{q} \rightarrow 1$ and the $\mathrm{D} \rightarrow \infty$ limits are to be taken simultaneously on both sides of equation (69). In this limit the phase operator $\hat{\mathcal{E}}_{\phi}$ is the unitary version of the Hermitian Pegg-Barnett phase operator [29]. Making use of the fact that $\hat{Q}=q^{\hat{N}}$, equation (67) yields the SusskindGlogower-Carruthers-Nieto commutation relations $[5,7,15]$ for the operator pair $\left(\hat{N}, \hat{\mathcal{E}}_{\phi}\right)$. Note that, the $\mathrm{QHO}$ described by $\mathrm{H}=\omega$ n yields the equation of motion for the phase operator in equation (69) which is formally identical to the equation of motion of the canonical angle variable for the classical harmonic oscillator. When we examine the quantum AA formalism of the generalized quantum oscillator in section 4.2, the harmonic oscillator will be realized 
in the specific limit when the dimension of the discrete phase space representations of the generalized oscillator is extended to infinity.

\section{An equivalent realization of the Wigner function by $\left(\mathrm{G}^{\wedge}, \mathrm{O}^{\wedge}\right)$}

\subsection{A generalized approach to the AA Wigner function using continuous phase space representations}

It was shown that the generalized canonical phase space representation of a quantum system can be given based on the duality between the discrete WK and the unitary cyclic Schwinger operator bases $[15,16]$ in equation (1). An alternative to this approach is to formulate the same problem using the canonical transformation $\mathrm{G}^{\wedge}$ and its unitary canonical partner $\mathrm{O}^{\wedge}$.

The properties of $(\hat{\mathcal{G}}, \hat{\mathcal{O}})$ studied in sections 2 and 3 manifest a full analogy to those of $(\hat{\mathcal{U}}, \hat{\mathcal{V}})$ in equations (3)-(5). Among the four equivalent choices in (5), we define this analogy by the correspondence

$$
\left(\begin{array}{l}
\hat{\mathcal{U}} \\
\hat{\mathcal{V}}
\end{array}\right) \Leftrightarrow\left(\begin{array}{l}
\hat{\mathcal{O}} \\
\hat{\mathcal{G}}
\end{array}\right) .
$$

It is now suggestive to define a Schwinger operator basis labelled by $\tau \mathrm{E}=\left(\tau_{1}, \tau_{2}\right) \in \mathrm{R} \times \mathrm{R}$ and defined as

$$
6^{\wedge}{ }_{\tau}=\mathrm{e}-\mathrm{i} \tau_{1} \tau / 2 / 2 \mathrm{O}^{\wedge}{ }_{\tau 1} \mathrm{G}^{\wedge}{ }_{\tau 2}=\mathrm{ei \tau} 1 \tau 2 / 2 \mathrm{G}^{\wedge}{ }_{\tau 2} \mathrm{O}^{\wedge}{ }_{\tau 1} \text {. }
$$

$\mathrm{E}$

Before we study the algebraic properties of $6^{\wedge} \tau \mathrm{E}$, we look into some of the tracial properties of $\mathrm{G}^{\wedge}$ and $\mathrm{O}^{\wedge}$ operators. Since we consider the continuum limit, it is more appropriate to examine $\mathrm{G}^{\wedge}$ and $\mathrm{O}^{\wedge}$ in their continuous representation. Starting with equation (39) we choose the $\mid \mathrm{h}(\gamma) \mathrm{i}$ basis for their representation as

$$
\hat{\mathcal{O}}^{\tau_{1}}=\int_{-\infty}^{\infty} \mathrm{d} \gamma\left|h^{\chi}\left(\gamma+\tau_{1}\right)\right\rangle\left\langle h^{\chi}(\gamma)\left|\quad \hat{\mathcal{G}}^{\tau_{2}}=\int_{-\infty}^{\infty} \mathrm{d} \gamma \mathrm{e}^{-\mathrm{i} \tau_{2} \gamma}\right| h^{\chi}(\gamma)\right\rangle\left\langle h^{\chi}(\gamma)\right|
$$

from which we obtain

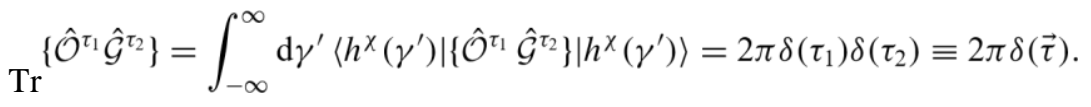

Using equation (73) the properties of $6^{\wedge} \tau \mathrm{E}$ can be found in manifest analogy with those of $\mathrm{S}^{\wedge} \mathrm{mE}$ in equation (7) as

$$
\begin{aligned}
& 6^{\wedge} \tau^{\dagger} \mathrm{E}=6^{\wedge}-\tau \mathrm{E} \\
& 6 \operatorname{Tr}^{\wedge} \tau \mathrm{E}\left\{66^{\wedge}{ } \mathrm{E} \tau \mathrm{E} 0_{0}\right\}=\mathrm{e} 2^{\mathrm{i}}{ }_{\tau \mathrm{E}} \pi \delta\left({ }^{\left.\times \mathrm{E}_{\tau 0} / \tau\right) \mathrm{E} 26^{\wedge}{ } \mathrm{E}+\tau \mathrm{E} 00}\right. \\
& \left(6^{\wedge} \tau \mathrm{E} 6^{\wedge} \tau \mathrm{E}_{0}\right) 6^{\wedge} \tau \mathrm{E}_{00}=6^{\wedge} \tau \mathrm{E}\left(6^{\wedge}{ } \mathrm{E}_{0} 6^{\wedge} \tau \mathrm{E}_{00}\right) \quad \text { (associativity) }
\end{aligned}
$$


$6^{\wedge} \mathrm{OE}=\mathrm{I}$ (unit element) 6^ $\tau \mathrm{E} 6^{\wedge}-\tau \mathrm{E}=\mathrm{I}$ (inverse). 
Hence, the canonical transformation generator $G^{\wedge}$ and its unitary canonical partner $\hat{O}$ form a continuous realization of Schwinger's operator basis.

Using equation (1) and the analogy manifested by equation (70) we construct a dual form for the WK operator basis using the realization of the Schwinger basis defined in equations (71) and (74) as

$$
\hat{\Delta}_{C T}(\vec{V})=\int \frac{\mathrm{d} \vec{\tau}}{2 \pi} \mathrm{e}^{-\mathrm{i} \vec{\tau} \times \vec{V}} \hat{\Sigma}_{\vec{\tau}} \quad \hat{\Sigma}_{\vec{\tau}}=\int \frac{\mathrm{d} \vec{V}}{2 \pi} \mathrm{e}^{\mathrm{i} \vec{\tau} \times \vec{V}} \hat{\Delta}_{C T}(\vec{V})
$$

where the integrals are to be considered in $\mathrm{R} \times \mathrm{R}$. Using the properties of $\hat{\Sigma}_{\bar{\tau}}$ it can be shown that equations (75) provide an operator basis for the Wigner function as

$$
\begin{aligned}
& \hat{\Delta}_{C T}(\vec{V})=\hat{\Delta}_{C T}^{\dagger}(\vec{V}) \\
& \int \frac{\mathrm{d} \vec{V}}{2 \pi} \hat{\Delta}_{C T}(\vec{V})=\mathbb{I} \\
& \operatorname{Tr}\left\{\hat{\Delta}_{C T}(\vec{V})\right\}=\mathbb{I} \\
& \operatorname{Tr}\left\{1^{\wedge} \mathrm{CT}(\mathrm{V}) \mathrm{E} 1^{\wedge} \mathrm{CT}\left(\mathrm{VE}^{0}\right)\right\}=\delta\left(\mathrm{VE}-\mathrm{VE}^{0}\right) .
\end{aligned}
$$

The properties (76) are necessary and sufficient conditions in order to define a correspondence between an arbitrary operator $\mathrm{F}^{\wedge}$ and its WWM symbol $\mathrm{f}(\mathrm{V}) \mathrm{E}$

$$
\mathrm{F}^{\wedge}=\mathrm{ZdV} f(\mathrm{EV}) \mathrm{E} 1^{\wedge} \text { ст }(\mathrm{V}) \mathrm{E} \mathrm{f}(\mathrm{V}) \mathrm{E}=\operatorname{Tr}\left\{\mathrm{F}^{\wedge} 1^{\wedge} \mathrm{cт}(\mathrm{V}) \mathrm{E}\right\}
$$

with the condition that $3 \mathrm{kF}^{\wedge} \mathrm{k}=\mathrm{R} \mathrm{dVE}|\mathrm{f}(\mathrm{V}) \mathrm{E}|^{2}<\infty$. A few simple examples can be given:3

(a) for $\mathrm{F}^{\wedge}=\mathrm{G}^{\wedge}$.

Using the same relations as in (a), the WWM symbol of $\mathrm{G}^{\wedge}$ is

$$
\mathrm{g}_{3}(\mathrm{~V}) \mathrm{E}=\operatorname{Tr}\left\{\mathrm{G}^{\wedge} 1^{\wedge} \mathrm{CT}(\mathrm{V}) \mathrm{E}\right\}=\mathrm{e}^{-\mathrm{i} 3 \mathrm{~V}_{1}}
$$

and similarly

(b) for $\mathrm{F}^{\wedge}=\mathrm{O}^{\wedge}$

$$
\mathrm{O}_{\zeta}(\mathrm{V}) \mathrm{E}=\operatorname{Tr}\left\{\mathrm{O}^{\wedge \zeta} 1^{\wedge} \mathrm{CT}(\mathrm{V}) \mathrm{E}\right\}=\mathrm{e}^{\mathrm{i} \zeta \mathrm{V}_{2}} .
$$

(c) A particularly interesting case arises when the arbitrary operator $\mathrm{F}^{\wedge}$ is invariant under a specific unitary transformation by $6^{\wedge} \mu \mathrm{E}$ such that 
$\hat{\Sigma}_{-\vec{\mu}} \hat{F} \hat{\Sigma}_{\vec{\mu}}=\hat{F}$. (80) Such an operator has a translationally invariant WWM symbol

$$
f(V) E=f(V E+\mu) E
$$

where, since 3 is arbitrary, it is implied that $\mathrm{f}(\mathrm{V}) \mathrm{E}$ is independent of $\mathrm{VE} \cdot \mathrm{En}_{\mu}$

where is the unit vector in the $\mu \mathrm{E}$ direction.

For $\mathrm{G}^{\wedge}$ describing the elements of the dynamical symmetry group, $1_{\mathrm{CT}}(\mathrm{V}) \mathrm{E}$ corresponds to thequantumAAoperatorrepresentationoftheWKbasis. Basedontheanalogyinequation(70) and the generalized Wigner function defined in equation (8) we now define a generalized $A A$ Wigner function of an arbitrary quantum state $\mid \psi i$ as

$$
\mathrm{W}_{\psi}(\mathrm{V}) \mathrm{E}=\mathrm{h} \psi\left|1_{\mathrm{CT}}(\mathrm{V}) \mathrm{E}\right| \psi \mathrm{i}
$$

which can be expressed in the continuous action eigenbasis as

$$
W_{\psi}(\vec{V})=\int_{-\infty}^{\infty} \frac{\mathrm{d} \tau_{1}}{2 \pi} \mathrm{e}^{\mathrm{i} \tau_{1} V_{2}}\left\langle\psi \mid h^{\kappa}\left(V_{1}-\tau_{1} / 2\right)\right\rangle\left\langle h^{\kappa}\left(V_{1}+\tau_{1} / 2\right) \mid \psi\right\rangle
$$

and in the continuous angle eigenbasis as

$$
W_{\psi}(\vec{V})=\int_{-\infty}^{\infty} \frac{\mathrm{d} \tau_{2}}{2 \pi} \mathrm{e}^{-\mathrm{i} \tau_{2} V_{1}}\left\langle\psi \mid k^{\chi}\left(V_{2}-\tau_{2} / 2\right)\right\rangle\left\langle k^{\chi}\left(V_{2}+\tau_{2} / 2\right) \mid \psi\right\rangle .
$$

Thus, equations (8) and (82) are two equivalent phase space representations of the same quantum system. The former is defined in a generic canonical basis $\left(\mathrm{U}^{\wedge}, \mathrm{V}^{\wedge}\right)$, whereas the latter is expressed in terms of the elements of the dynamical symmetry group of the same system. In equations (83) and (84) $\mathrm{V}_{1}$ and $\mathrm{V}_{2}$ are, by the WWM correspondence in equations (78) and (79), the generalized classical AA variables.

\subsection{AA Wigner function for the generalized oscillator with the discrete phase space representations}

In the previous sections we examined the generalized theory of quantum AA formalism using the continuous representations of the generalized AA operators. Here, we particularize this formalism to that of the representations of a generalized oscillator with a discrete spectrum in the finite $\mathrm{D}$-dimensional Hilbert space $\mathrm{H}_{\mathrm{D}}$ by constructing the unitary canonical pair $\left(\mathrm{G}^{\wedge}, 0^{\wedge}\right)$ as

$$
\left(\begin{array}{l}
\hat{\mathcal{G}} \\
\hat{\mathcal{O}}
\end{array}\right) \Leftrightarrow\left(\begin{array}{l}
\hat{\mathcal{E}}_{N} \\
\hat{\mathcal{E}}_{\phi}
\end{array}\right)
$$

where $\hat{\mathcal{E}}_{N}=\mathrm{e}^{-\mathrm{i} \gamma_{0} \hat{N}}$ with $\hat{N}_{\text {as }}$ defined in equations (A.3), (A.4) and (64). The unitary operator

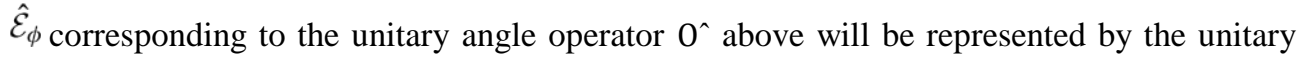
quantum phase operator defined in equations (66)-(68). To facilitate the correspondence with the classical case, we switch from the generalized notation $\mathrm{VE}=\left(\mathrm{V}_{1}, \mathrm{~V}_{2}\right)$ of the AA variables in section 4.1 to the more standard one $(\mathrm{J}, \theta)$. The realization of the AA WK basis in the unitary number-phase basis $\left(\hat{\mathcal{E}}_{N}, \hat{\mathcal{E}}_{\phi}\right)$ has been derived in [15] for the generalized oscillator with 
Linear canonical transformations and quantum phase

discrete, cyclic and finite D-dimensional Hilbert space representations as $\hat{\Delta}_{C T}(J, \theta)=\frac{1}{2 \pi D} \sum_{\vec{m}} \mathrm{e}^{\mathrm{i}\left(\gamma_{0} m_{1} J-m_{2} \theta\right)} \mathrm{e}^{-\mathrm{i} \gamma_{0} m_{1} m_{2} / 2} \hat{\mathcal{E}}_{N}^{m_{1}} \hat{\mathcal{E}}_{\phi}^{m_{2}} \mathrm{mE} \in \mathrm{ZD} \times \mathrm{ZD}_{\mathrm{D}}$ (86)

In the discrete case, the set of completeness relations analogous to the continuous ones in equation (76) — by direct use of equation (86) — are

$$
\begin{aligned}
& \hat{\Delta}_{C T}(J, \theta)=\hat{\Delta}_{C T}^{\dagger}(J, \theta) \\
& \int \mathrm{d} J \int \mathrm{d} \theta \hat{\Delta}_{C T}(J, \theta)=\mathbb{I} \\
& \operatorname{Tr}\left\{\Delta_{C T}(J, \theta)\right\}=\frac{1}{2} \pi \\
& \operatorname{Tr}\left\{\hat{\Delta}_{C T}(J, \theta) \hat{\Delta}_{C T}\left(J^{\prime}, \theta^{\prime}\right)\right\}=\frac{1}{2} \pi \delta\left(J-J^{\prime}\right) \delta\left(\theta-\theta^{\prime}\right) . \\
& \hat{\Delta}_{C T}
\end{aligned}
$$

The AA Wigner function of $\quad D(\mathrm{~J}, \theta)$ in a physical state $\mid \psi \mathrm{i}$ is then given by [15]

$W(J, \theta)=\left\langle\psi\left|\hat{\Delta}_{C T}(J, \theta)\right| \psi\right\rangle=\frac{1}{2 \pi} \sum_{k=0}^{-1} \mathrm{e}^{-\mathrm{i} k \theta}\langle\psi \mid J-k / 2\rangle\langle J+k / 2 \mid \psi\rangle \quad k \in \mathbb{Z}$

where the states $\mid \mathrm{J} \pm \mathrm{k} / 2 \mathrm{i}$ are vectors in continuously shifted Fock spaces [15]. Here $\{\mid \mathrm{J} \pm \mathrm{k} / 2 \mathrm{i} ; \mathrm{k}$ $=\operatorname{odd}\} \in \mathrm{F}^{(\alpha \pm 1 / 2)}$ and $\{\mid \mathrm{J} \pm \mathrm{k} / 2 \mathrm{i} ; \mathrm{k}=$ even $\} \in \mathrm{F}^{(\alpha)}$ with $\alpha$ satisfying the conditions [15] that $2(\mathrm{~J}-$ $\alpha) \in \mathrm{Z}$ and $(\beta) \alpha \in \mathrm{R}[0,1)$. The definition of an arbitrary vector in the continuously shifted Fock space $\mathrm{F}$ has been given in [15] by

$$
|n+\beta\rangle \equiv \frac{1}{\sqrt{D}} \sum_{\ell=0}^{D-1} \mathrm{e}^{-\mathrm{i} \gamma_{0}(n+\beta) \ell}|\phi\rangle_{\ell} \quad \beta \in \mathbb{R}[0,1) \quad|n+\beta\rangle \equiv|n+D+\beta\rangle \in \mathcal{F}^{(\beta)} .
$$

Now, let us take D $\rightarrow \infty$ in equation (86) and, using equation (89) examine equation (88) for the type $\mid \psi$ i being: (a) a pure Fock state, i.e. $\left|\psi \mathrm{im}=(|\mathrm{ni}+| \mathrm{n}-1 \mathrm{i}) / \sqrt{ } 2_{-},\right| \psi \mathrm{n}$ ifixed.p $=\mid$ ni, $\mathrm{n}$ fixed

$$
\text { and (b) a typical mixed Fock state of }
$$

(a) For $\mid \psi$ ip, and after a short calculation, equation (88) can be evaluated in the limit $\mathrm{D} \rightarrow \infty$ as

$$
\left.W(J, \theta)\right|_{p}=\frac{1}{2 \pi} \delta(n-J) .
$$

The marginal probability distributions for the $\mathbf{J}$ or $\theta$ variables in the pure Fock state can then be found by integrating over the other variable $\theta$ or $\mathrm{J}$, respectively, as

$$
\begin{aligned}
\left.P(J)\right|_{p} & \equiv \int \mathrm{d} \theta W_{p}(J, \theta)=\delta(n-J) \\
\left.\tilde{P}(\theta)\right|_{p} & \equiv \int \mathrm{d} J W_{p}(J, \theta)=\frac{1}{2 \pi}
\end{aligned}
$$

which correctly describe the expected results for the pure Fock state. 
(b) The state $/ \psi \mathrm{im}$ is the so-called split state. For this state, using equation (89), we obtain

$$
\left.W(J, \theta)\right|_{m}=\frac{1}{4 \pi}\{\delta(n-J)+2 \delta(n-J-1 / 2) \cos \theta+\delta(n-J-1)\} .
$$

The marginal probability distributions yield for $\mid \psi i \mathrm{~m}$

$$
\begin{aligned}
& \left.P(J)\right|_{m}=\{\delta(n-J)+\delta(n-J-1)\} / 2 \\
& \left.\tilde{P}(\theta)\right|_{m}=(1+\cos \theta) / 2 \pi
\end{aligned}
$$

which are the correct action and angle probability distributions for the split state.

Equation (88) also provides the correct time dependence for the AA Wigner function in the QHOlimit. Inordertoobservethiswewillstartwiththegeneralizedoscillatorinequation(63). The time dependence of the AA Wigner function is given by the standard expression

$$
\begin{aligned}
& \text { d } \\
& \text { id_t } \mathrm{W}_{\psi}(\mathrm{J}, \theta)=\mathrm{h} \psi\left|\left[\mathrm{H}^{\wedge}, 1^{\wedge}{ }^{\wedge} \mathrm{CT}(\mathrm{J}, \theta)\right]\right| \psi \mathrm{i}
\end{aligned}
$$

or, equivalently, in terms of the WWM symbol $\mathrm{h}(\mathrm{J}, \theta)$ of $\mathrm{H}^{\wedge}$ as [30]

$$
\begin{aligned}
& W_{\psi}(J, \theta)=\left\{h(J, \theta) * W_{\psi}(J, \theta)-W_{\psi}(J, \theta) * h(J, \theta)\right\} \\
& \underline{\mathrm{d}} \\
& \mathrm{dt} *=\exp \frac{\mathrm{i}}{2} \frac{\partial}{\partial J} \frac{\partial}{\partial \theta}-\frac{\partial}{\partial \theta} \frac{\partial}{\partial J}\left(\begin{array}{lll} 
& \mathrm{E} & \mathrm{E} \#)
\end{array}\right.
\end{aligned}
$$

where $\left(\mathrm{h} * \mathrm{~W}_{\psi}-\mathrm{W}_{\psi} * \mathrm{~h}\right) \equiv\left\{\mathrm{h}, \mathrm{W}_{\psi}\right\}_{\text {MB }}$ is the Moyal (sine) bracket [30]. The calculation of equation (95) requires the knowledge of $\mathrm{h}(\mathrm{J}, \theta)$. This can be obtained by using the completeness equations in (87) and the Hamiltonian operator $\mathrm{H}^{\wedge}$ in (63) as

$\mathrm{H}^{\wedge}=\mathrm{Z} \mathrm{dJ} Z \mathrm{~d} \theta \mathrm{h}(\mathrm{J}, \theta) 1^{\wedge} \mathrm{Cт}(\mathrm{J}, \theta) \quad$ where $\quad \mathrm{h}(\mathrm{J}, \theta)=\operatorname{Tr}\left\{\mathrm{H}^{\wedge} 1_{\mathrm{CT}}(\mathrm{J}, \theta)\right\}$.

The trace operation can be conveniently carried in the discrete finite-dimensional cyclic eigenspace $\{\mid \mathrm{ni}\}=\left\{\left|\operatorname{ni}_{6 \mathrm{n} 6\left(\mathrm{D}^{-1}\right)} ;\right| \mathrm{n}+\mathrm{Di} \equiv \mid \mathrm{ni}\right\}$ of the Hamiltonian $\mathrm{H}^{\wedge}$. Since for the diagonal matrix elements $\mathrm{hn}\left|1^{\wedge} \mathrm{CT}(\mathrm{J}, \theta)\right| \mathrm{ni}=\mathrm{W}(\mathrm{J}, \theta) \mid \mathrm{p}$ are Wigner functions of the pure Fock states, we can also directly use the expression (90) in the calculation of the trace. We find that

$$
\mathrm{h}(\mathrm{J}, \theta)=\mathrm{H}(\mathrm{J}) \text {. }
$$

Using equation (97) in equation (95)

$$
\underset{\mathrm{d}}{\mathrm{d}} \mathrm{W}_{\psi}(\mathrm{J}, \theta)=-\mathrm{i}\left\{\mathcal{H}\left(J+\frac{\mathrm{i}}{2} \frac{\partial}{\partial \theta}\right)-\mathcal{H}\left(J-\frac{\mathrm{i}}{2} \frac{\partial}{\partial \theta}\right)\right\} W_{\psi} \quad(\mathrm{J}, \theta) .
$$


Equation (98) is the equation of motion of the AA Wigner function for an arbitrary generalized cyclic oscillator in equation (63). Now, we apply equation (98) to the QHO case where the Hamiltonian in equation (63) is a linear operator of $\mathrm{N}^{\wedge}$, let us say $\mathrm{H}(\mathrm{N})^{\wedge}=\omega \mathrm{N}^{\wedge}$ with $\omega$ describing the oscillator frequency. Then, by equations (96) and $(97), h(J, \theta)=\omega J$. For the QHO equation (98) then yields,

$$
\left\{\frac{\mathrm{d}}{\mathrm{d} t}-\omega \frac{\partial}{\partial \theta}\right\} W_{\psi}(J, \theta)=0 \quad \mathrm{H} \Rightarrow \quad \theta=\theta(\mathrm{t})=\omega \mathrm{t}
$$

namely, the time evolution of the QHO AA Wigner function in the phase space takes place on the classical manifold $(\mathrm{J}=$ constant, $\theta=\omega \mathrm{t})$ as expected. By equation (90), The AA Wigner function for the pure Fock state is static. The full time dependent solution of the AA Wigner function, for instance, for the split state and the corresponding marginal probability distributions can be completely determined by inserting the solution of $\theta(t)$ in equation (99) into equations (92) and (93).

\section{Conclusions}

The canonical-algebraic connection between the quantum phase problem and the QPS has already been noticed recently by some other workers. In particular, using the generators of the angular momentum su(2) algebra and its dual in terms of the Hermitian canonical phase operators Vourdas studied [31] an equivalent canonical pair to $\left(\mathrm{G}^{\wedge}, \mathrm{O}^{\wedge}\right)$ as defined in this paper. Our specific aim in this paper was to further the canonical algebraic approach introduced in [15] to unify the formulation of quantum phase with that of the algebraic theory of quantum canonicaltransformations. Inthiscontext, weinvestigatedthegeneratorsofquantumcanonical transformations, their unitary canonical partners in the Schwinger sense, as well as their action on the functions of canonical variables of the QPS, in particular the Wigner function. Through this connection, the quantum phase is formally established as the unitary canonical partner of the quantum action operator which is also demonstrated for the one-dimensional generalized oscillator.

\section{Acknowledgments}

The author is grateful to Dr Laurence Barker (Bilkent University) for illuminating discussions, particularly on the topics covered in section 2.1. Stimulating discussions with Professor T Dereli (Middle East Technical University) and Professor M Arik (Bogaziçi־ University) are also acknowledged.

\section{Appendix A.}

In [15] we examined two subalgebraic realizations of the discrete-cyclic Schwinger operator basis $\hat{\mathcal{S}}_{\vec{m}}$. In the following we will have a brief summary of them. Based on a fixed pair of vectors $\mathrm{m}, \mathrm{E} \mathrm{mE}^{0}$ the sine algebra generated by $\hat{S}_{\vec{m}}$ supports two subalgebraic realizations [15]. 4129 The standard $\mathrm{u}_{\mathrm{q}} \mathrm{sl}(2)$ subalgebraic realization is obtained by constructing the generators 


$$
\begin{aligned}
& \text { T Hakioglu } \\
& \hat{J}_{-} \equiv d \hat{S}_{\vec{m}}+d^{\prime} \hat{S}_{\vec{m}^{\prime}} \\
& \hat{J}_{+} \equiv d^{*} \hat{S}_{-\vec{m}}+d^{\prime *} \hat{S}_{-\vec{m}^{\prime}} \\
& \hat{L} \equiv \hat{S}_{\vec{m}-\vec{m}^{\prime}}=q^{\hat{J}_{3}+\frac{D}{2}}
\end{aligned}
$$

where $\mathrm{dd} 0 *=\mathrm{d} * \mathrm{~d} 0=-(\mathrm{q} 1 / 2-\mathrm{q}-1 / 2)-2$ and $\mathrm{q}=\mathrm{e}-\mathrm{i} \mathrm{m}_{0} \mathrm{mE} \times$ Emo so that

$\mathrm{q}^{ \pm 1} \mathrm{~L}^{\wedge} \mathrm{J}^{\wedge} \bar{\mp}$

$$
\mathrm{J}^{\wedge} \mathrm{L}^{\wedge}=\quad\left[\hat{J}_{-}, \hat{J}_{+}\right]=-\frac{L-L^{-1}}{q^{1 / 2}-q^{-1 / 2}}=-\left[\hat{J}_{3}+\frac{D}{2}\right]
$$

$\mp$

This particular subalgebraic realization is sometimes referred as the magnetic translation group [32].

On the other hand, more importantly for the purpose of this work, a second class of subalgebraic realizations exist in the form of an admissible q-oscillator algebra which can be obtained by defining

$$
\begin{aligned}
& \mathrm{A}^{\wedge} \equiv \mathrm{dS}^{\wedge} \mathrm{m}_{\mathrm{E}}+\mathrm{d}^{0} \mathrm{~S}^{\wedge} \mathrm{m}_{\mathrm{E} 0} \\
& \mathrm{~A}^{\wedge \uparrow} \equiv \mathrm{d}^{*} \mathrm{~S}^{\wedge}{ }_{-\mathrm{mE}}+\mathrm{d}^{0} * \mathrm{~S}^{\wedge}{ }_{-\mathrm{mE} 0} \\
& -\vec{m}-\vec{m} \quad \hat{\mathrm{Q}} \equiv \mathrm{q}-\mathrm{N}^{\wedge}-(\mathrm{D}-1) / 2=\mathrm{q} 1 / 2 \mathrm{~S}^{\wedge}(0) \\
& d d^{*}=-d^{*} d^{\prime}=-\left(q-q^{-1}\right)^{-1} \text { and } q=\mathrm{e}^{-\mathrm{i} \gamma_{0} \vec{m} \times \vec{m}^{\prime}} \\
& \hat{A}^{\dagger} \hat{A}=C+[\hat{N}] \\
& \mathrm{A}^{\wedge} \mathrm{Q}^{\wedge}=\mathrm{q}^{-} 1 \mathrm{Q}^{\wedge} \mathrm{A}^{\wedge} \mathrm{A}^{\wedge} \dagger \mathrm{Q}^{\wedge}=\mathrm{q} \\
& \mathrm{Q}^{\wedge} \mathrm{A}^{\wedge}
\end{aligned}
$$

such that

$$
\hat{A} \hat{A}^{\dagger}-q \hat{A}^{\dagger} \hat{A}=(1-q) C+\hat{Q}
$$

where $\left[\mathrm{N}^{\wedge}\right]=\left(\mathrm{Q}^{\wedge}-\mathrm{Q}\right) /\left(\mathrm{q}^{\wedge}-\mathrm{q}^{-1}\right)$ and $\mathrm{C}=\left(\mid \sin \left(\gamma 0 \mathrm{mE} \times \mathrm{Em}^{0}\right)\right)^{-1}$. Equations (A.4) imply that the q-oscillator spectrum is non-negative (i.e. $06 \mathrm{kA}^{\wedge \uparrow} \mathrm{A}^{\wedge} \mathrm{k}$ where the spectrum is given by the eigenvalues of the operator $\mathrm{A}^{\wedge \uparrow} \mathrm{A}^{\wedge}=\mathrm{C}+\left[\mathrm{N}^{\wedge}\right]$ ) which, further implies that the Hilbert space is spanned by vectors with admissible (non-negative) norm. It was shown in [15] that the admissible q-oscillator algebra in equations (A.3) and (A.4) is crucial in establishing a canonical-algebraic approach to the quantum phase problem. Interested readers can find more detailed discussions of the admissible q-oscillator realizations therein.

\section{References}

[1] Born M, Heisenberg W and Jordan P 1926 Z. Phys. 35557

[2] Dirac P A M 1927 Proc. R. Soc. A 114243

Dirac P A M 1958 Principles of Quantum Mechanics (Oxford: Oxford University Press)

[3] Heitler W 1958 The Quantum Theory of Radiation (London: Academic)

[4] Louisell W H 1963 Phys. Lett. 760 
Linear canonical transformations and quantum phase

[5] Susskind L and Glogower J 1964 Physics 149 [6] Glauber R J 1963 Phys. Rev. 1302529

Glauber R J 1963 Phys. Rev. 1312766

[7] Carruthers P and Nieto M M 1968 Rev. Mod. Phys. 40411

Carruthers P and Nieto M M 1965 Phys. Rev. Lett. 14387

[8] Tanas R, Miranowicz A and Gantsog Ts 1996' Prog. Opt. 35355

Pegg D T and Barnett S M 1997 J. Mod. Opt. 44225

[9] Mandel L and Wolf E 1995 Optical Coherence and Quantum Optics (Cambridge: Cambridge University Press) Vogel W and Welsh D-G 1994 Quantum Opt. (Berlin: Akademia)

[10] Rocca F and Siruge M 1973 Commun. Math. Phys. 34111 [11] Boyer C P and Wolf K B 1974 J. Math. Phys. 161493 Wolf K B 1974 J. Math. Phys. 152102

[12] Moshinsky M and Seligman T H 1978 Ann. Phys., NY 114243

Moshinsky M and Seligman T H 1979 J. Phys. A: Math. Gen. 12 L135

[13] Luis A and Sanchez-Soto L L 1993 Phys. Rev. A 48752

[14] Lewis H R, Lawrence W E and Harris J D 1996 Phys. Rev. Lett. 775157

[15] Hakioglu T 1998 J. Phys. A: Math. Gen. 316975 [16] Schwinger J 1960 Proc. Natl Acad. Sci. 46 883

Schwinger J 1960 Proc. Natl Acad. Sci. 461401

[17] Galetti D and de Toledo Piza A F R 1988 Physica A 149267 Aldrovandi R and Galetti D 1990 J. Math. Phys. 312987

[18] Wigner E P 1932 Phys. Rev. 40749

Kirkwood J G 1933 Phys. Rev. 4431

[19] Fairlie D, Fletcher P and Zachos C 1989 Phys. Lett. B 218203 Fairlie D B and Zachos C 1989 Phys. Lett. B 224101

[20] Arnold V 1966 Ann. Inst. Fourier 16319

Arnold V 1978 Mathematical Methods of Classical Mechanics (Berlin: Springer)

[21] Balian R and Itzykson C 1986 C. R. Acad. Sci., Paris 303773

Athanasiu G G and Floratos E G 1994 Nucl. Phys. B 425343

[22] Weyl H 1931 The Theory of Groups in Quantum Mechanics (New York: Dover)

[23] Ellinas D and Floratos E G 1998 Prime decomposition and entanglement measure of finite quantum systems Preprint quant-ph/9806007

[24] Namias V 1980 J. Inst. Math. Appl. 25241

[25] Soo-Chang Pei and Min-Hung Yeh 1997 Opt. Lett. 221047

[26] Candan Ç, Alper Kutay M and Ozaktas H M 1999 The discrete fractional Fourier transformation Proc. IEEE Int. Conf. Acoustic, Speech and Signal Processing (Piscataway, NJ: IEEE) pp 1713-6

[27] Gilmore R 1974 Lie Groups, Lie Algebras and Some of Their Applications (New York: Wiley)

[28] Wang S J and Chu S Y 1994 J. Phys. A: Math. Gen. 275655 [29] Pegg D T and Barnett S M 1988 Europhys. Lett. 6483

Pegg D T and Barnett S M 1989 Phys. Rev. A 391665

[30] Moyal J 1949 Proc. Camb. Phil. Soc. 4599 [31]

Vourdas A 1990 Phys. Rev. A 411653

Vourdas A 1996 J. Phys. A: Math. Gen. 294275

Vourdas A 1991 Phys. Rev. A 431564

Vourdas A and Bendjaballah C 1993 Phys. Rev. A 473523

[32] Zak J 1964 Phys. Rev. 1341602

Brown E 1964 Phys. Rev. 1331038

Shen X 1992 Int. J. Mod. Phys. A 73717

Dereli T and Verçin A 1992 Phys. Lett. B 288109

Kogan I 1994 Int. J. Mod. Phys. A 93887 